\title{
Article \\ Correlation of Body Mass Index with Oncologic Outcomes in Colorectal Cancer Patients: A Large Population-Based Study
}

\author{
Chong-Chi Chiu ${ }^{1,2, \dagger}$, Chung-Han Ho ${ }^{3,4,5,+}$, Chao-Ming Hung ${ }^{1,6}$, Chien-Ming Chao ${ }^{7,8}$, Chih-Cheng Lai ${ }^{9}{ }^{\circledR}$,

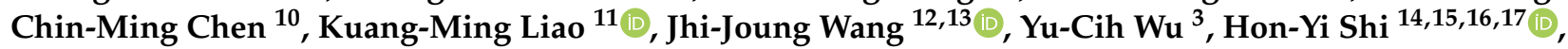 \\ Po-Huang Lee ${ }^{6,18}$, Hui-Ming Lee ${ }^{1,6}$, Li-Ren Yeh ${ }^{19,20}$, Tien-Chou Soong ${ }^{6,21}$, Shyh-Ren Chiang ${ }^{22,23, \mp}$ \\ and Kuo-Chen Cheng $22,24, *, \ddagger$
}

Citation: Chiu, C.-C.; Ho, C.-H.; Hung, C.-M.; Chao, C.-M.; Lai, C.-C.; Chen, C.-M.; Liao, K.-M.; Wang, J.-J.; Wu, Y.-C.; Shi, H.-Y.; et al. Correlation of Body Mass Index with Oncologic Outcomes in Colorectal Cancer Patients: A Large Population-Based Study. Cancers 2021, 13, 3592. https://doi.org/10.3390/ cancers13143592

Academic Editors: Luciano Cascione, Francesco Denti and Antonietta Mira

Received: 5 May 2021

Accepted: 14 July 2021

Published: 17 July 2021

Publisher's Note: MDPI stays neutral with regard to jurisdictional claims in published maps and institutional affiliations.

Copyright: (c) 2021 by the authors. Licensee MDPI, Basel, Switzerland. This article is an open access article distributed under the terms and conditions of the Creative Commons Attribution (CC BY) license (https:// creativecommons.org/licenses/by/ $4.0 /)$
1 Department of General Surgery, E-Da Cancer Hospital, Kaohsiung 82445, Taiwan; chiuchongchi@gmail.com (C.-C.C.); ed100647@edah.org.tw (C.-M.H.); huiming00@gmail.com (H.-M.L.)

2 School of Medicine, College of Medicine, I-Shou University, Kaohsiung 82445, Taiwan

3 Department of Medical Research, Chi Mei Medical Center, Tainan 71004, Taiwan; ho.c.hank@gmail.com (C.-H.H.); cih830927@gmail.com (Y.-C.W.)

4 Cancer Center, Wan Fang Hospital, Taipei Medical University, Taipei 11695, Taiwan

5 Department of Information Management, Southern Taiwan University of Science and Technology, Tainan 71005, Taiwan

6 College of Medicine, I-Shou University, Kaohsiung 82445, Taiwan; pohuang1115@ntu.edu.tw (P.-H.L.); tienchou0117@gmail.com (T.-C.S.)

7 Department of Intensive Care Medicine, Chi Mei Medical Center, Liouying 73657, Taiwan; ccm870958@yahoo.com.tw

8 Department of Dental Laboratory Technology, Min-Hwei College of Health Care Management, Tainan 73657, Taiwan

9 Department of Internal Medicine, Kaohsiung Veterans General Hospital, Tainan Branch, Tainan 71004, Taiwan; dtmed141@gmail.com

10 Department of Intensive Care Medicine, Chi-Mei Medical Center, Tainan 71004, Taiwan; chencm3383@yahoo.com.tw

11 Department of Internal Medicine, Chi Mei Medical Center, Chiali 72263, Taiwan; abc8870@yahoo.com.tw

12 Department of Anesthesiology, Chi Mei Medical Center, Tainan 71004, Taiwan; 400002@mail.chimei.org.tw

13 Department of Anesthesiology, National Defense Medical Center, Taipei 11490, Taiwan

14 Department of Healthcare Administration and Medical Informatics, Kaohsiung Medical University, Kaohsiung 80708, Taiwan; hshi@kmu.edu.tw

15 Department of Business Management, National Sun Yat-Sen University, Kaohsiung 80424, Taiwan

16 Department of Medical Research, Kaohsiung Medical University Hospital, Kaohsiung 80756, Taiwan

17 Department of Medical Research, China Medical University Hospital, China Medical University, Taichung 40402, Taiwan

18 Department of Surgery, E-Da Hospital, Kaohsiung 82445, Taiwan

19 Department of Anesthesiology, E-Da Cancer Hospital, Kaohsiung 82445, Taiwan; ed110880@edah.org.tw

20 Department of Medical Imaging and Radiology, Shu-Zen Junior College of Medicine and Management, Kaohsiung 82144, Taiwan

21 Weight Loss and Health Management Center, E-Da Dachang Hospital, Kaohsiung 80794, Taiwan

22 Department of Internal Medicine, Chi Mei Medical Center, Tainan 71004, Taiwan; crsmed@yahoo.com.tw

23 Department of General Education, Chia Nan University of Pharmacy and Science, Tainan 71710, Taiwan

24 Department of Safety, Health and Environment, Chung Hwa University of Medical Technology, Tainan 71703, Taiwan

* Correspondence: kcg.cheng@gmail.com; Tel.: +886-933672369; Fax: +886-6-2828928

+ These authors contributed equally to this work.

$\ddagger$ These authors contributed equally to this work.

Simple Summary: Obesity is related to the rising risk of colorectal cancer (CRC). However, the impact of body mass index (BMI) on the oncologic prognosis of CRC patients remains unknown. Conflicting results regarding the relationship between BMI and CRC prognosis have been reported. Therefore, we conducted a nationwide retrospective study that examined the correlation of BMI at diagnosis with overall survival (OS), disease-free survival (DFS), and CRC-specific survival rates in CRC patients. We noted that an underweight status at diagnosis was related to higher mortality and recurrence rates, a decreased rate of OS, and a decreased CRC-specific survival rate compared with those for the normal weight patients. In contrast, overweight and class I or II obese patients 
had better OS, CRC-specific survival, and DFS rates than those in the normal weight category. Our findings suggest that weight loss in the immediate diagnosis period is unwarranted.

Abstract: It has been acknowledged that excess body weight increases the risk of colorectal cancer (CRC); however, there is little evidence on the impact of body mass index (BMI) on CRC patients' longterm oncologic results in Asian populations. We studied the influence of BMI on overall survival (OS), disease-free survival (DFS), and CRC-specific survival rates in CRC patients from the administrative claims datasets of Taiwan using the Kaplan-Meier survival curves and the log-rank test to estimate the statistical differences among BMI groups. Underweight patients $\left(<18.50 \mathrm{~kg} / \mathrm{m}^{2}\right)$ presented higher mortality $(56.40 \%)$ and recurrence $(5.34 \%)$ rates. Besides this, they had worse OS (aHR:1.61; 95\% CI: 1.53-1.70; $p$-value: < 0.0001) and CRC-specific survival (aHR:1.52; 95\% CI: 1.43-1.62; $p$-value: <0.0001) rates compared with those of normal weight patients $\left(18.50-24.99 \mathrm{~kg} / \mathrm{m}^{2}\right)$. On the contrary, CRC patients belonging to the overweight $\left(25.00-29.99 \mathrm{~kg} / \mathrm{m}^{2}\right)$, class I obesity $\left(30.00-34.99 \mathrm{~kg} / \mathrm{m}^{2}\right)$, and class II obesity $\left(\geq 35.00 \mathrm{~kg} / \mathrm{m}^{2}\right)$ categories had better OS, DFS, and CRC-specific survival rates in the analysis than the patients in the normal weight category. Overweight patients consistently had the lowest mortality rate after a CRC diagnosis. The associations with being underweight may reflect a reverse causation. CRC patients should maintain a long-term healthy body weight.

Keywords: colorectal cancer; body mass index; oncologic prognosis; overall survival; disease-free survival; colorectal cancer-specific survival

\section{Introduction}

Colorectal cancer (CRC) is among the leading causes of cancer death worldwide, with an approximate incidence of $0.02 \%$ [1]. There are about 1.2 million newly diagnosed CRC patients every year [2]. The increasing incidence of CRC in East Asian countries has been attributed to changes in diet and lifestyle and the popularization of cancer screening in recent decades [3].

Obesity involves excessive body fat accumulation and, therefore, it increases the risk of metabolic syndrome [4]. It is a significant health concern worldwide. This global pandemic of obesity increases the risks of multiple cancer occurrence, including CRC [5]. Besides this, the 5-year survival rate of CRC is still disappointing, especially in developing areas [6]. Indeed, it is reasonable to pay attention to CRC prevention.

The body mass index (BMI) is the most popular scale to assess obesity [7]. A metaanalysis study including 41 reports found that obesity increased CRC risk by $33 \%$ among $8,115,689$ people [8]. Baade et al. [9] and Kuiper et al. [10] demonstrated that overweight CRC patients had improved CRC-related mortality rates by $25 \%$ and $55 \%$, respectively. However, Meyerhardt et al. showed no disparity in the mortality risk between overweight and normal weight CRC patients [11,12]. These conflicting results have also been found among studies that examined the correlation between obesity and CRC prognosis. Conversely, some studies have revealed that being underweight is related to an increased risk of mortality. This phenomenon is probably caused by cancer progression-associated weight loss [9,11-13]. Realizing the relationship between BMI and CRC prognosis is highly important to provide body weight guidelines for CRC patients [14].

Most studies about the relationship between obesity and a CRC oncologic prognosis were performed in European institutions and have seldom been performed in East Asian areas. Besides this, East Asian people generally have a lower ratio of overall obesity but are more centrally obese than Europeans. Some studies have demonstrated that Asian people have a comparatively higher visceral fat ratio than Europeans with the same BMI. Thus, Asian people are more vulnerable to insulin resistance [15]. From the literature, only four studies have investigated the impact of obesity on CRC oncologic prognoses among Asian people [16-19]. However, some demerits of these studies include the small sample sizes, the lack of investigation into the correlation between central obesity and 
CRC survival rates, and the lack of potential confounder adjustment [20]. This study was performed to investigate the clinical significance of BMI and oncologic outcomes using a large nationwide CRC patient cohort from Taiwan.

\section{Materials and Methods}

\subsection{Study Design and Data Sources}

The administrative claims datasets, including the national health insurance research database (NHIRD), the Taiwan Cancer Registry (TCR), and Taiwan's cause of death dataset, from the Health and Welfare Data Science Center (HWDC), Ministry of Health and Welfare (MOHW) were used in this study. The HWDC is a data center that integrates all healthrelated datasets for research purposes. The NHIRD is based on Taiwan's National Health Insurance program, which covers more than 23 million people. The NHIRD is comprised of detailed individual information, including age, sex, diagnosis date, disease and procedure codes, prescription, and reimbursement claims. Besides this, the TCR was built in 1979 to monitor Taiwan's cancer incidence, and the mortality rates were used to identify cancer patients. The basic information within the TCR includes patient demographics, clinical stages of cancer, cancer primary sites, tumor histology, and treatment types. To add more precise diagnosis and treatment items, in 2002 the TCR established long-form datasets for oncology categories such as colon and rectum [21].

The data for this study were retrieved from the TCR. The claims of diagnosis and the inpatient and outpatient information of patients were obtained from the NHIRD. Diagnosis codes were selected from the International Classification of Diseases, Ninth Revision, Clinical Modification (ICD-9-CM). This study was exempt from the institution's internal review board's full review because our secondary data analysis could not identify the patients. However, the study protocol conformed to the 1964 Declaration of Helsinki's ethical standards.

\subsection{Study Patients}

The TCR included all patients with information retrieved from the International Classification of Diseases for Oncology, third edition (ICD-O-3) with colon (ICD-O-3: C18), rectosigmoid junction (ICD-O-3: C19), and rectum (ICD-O-3: C20) as the primary cancer from January 2011 to December 2015. To exclude potential misclassification, the cases without a correct diagnosis date, information on the clinical stage, treatment type, body weight, or body height at the time of cancer diagnosis were excluded. A diagram illustrating the selection of study patients is provided in Figure 1.

\subsection{Measurements}

The study variables, including age at diagnosis, gender, clinical stage, histology grade, treatment type, and smoking status, were all collected from the TCR. The age group was divided into the categories $<40,40-49,50-59,60-69$, and $\geq 70$. Smoking status categories were defined as patients who have never smoked, have ever smoked (since quit), and currently smoke. As some comorbidities may result in premature mortality not related to the primary colon or rectum malignancies but may be closely associated with obesity, we also analyzed the effect of these comorbidities, including myocardial infarction (ICD-9-CM: 410, 412), congestive heart failure (ICD-9-CM: 428), peripheral vascular disease (ICD-9CM: 441, 443.9, 785.4), dementia (ICD-9-CM: 290,294, 331), chronic obstructive pulmonary disease (ICD-9-CM: 490-496, 500-505, 506.4), rheumatic disease (ICD-9-CM: 725, 710.0710.1, 710.4, 714.0-714.2, 714.81, 517.1), peptic ulcer disease (ICD-9-CM: 531-534), mild liver disease (ICD-9-CM: 571.2, 571.4-571.6), diabetes mellitus without chronic complications (ICD-9-CM: 250.0-250.3, 250.7), diabetes mellitus with chronic complications (ICD-9-CM: 250.4-250.6), hemiplegia or paraplegia (ICD-9-CM: 342, 344.1), renal disease (ICD-9-CM: 582-583, 585-586, 588), and moderate and severe liver diseases (ICD-9-CM: 456.0-456.2, 572.2-572.8). 


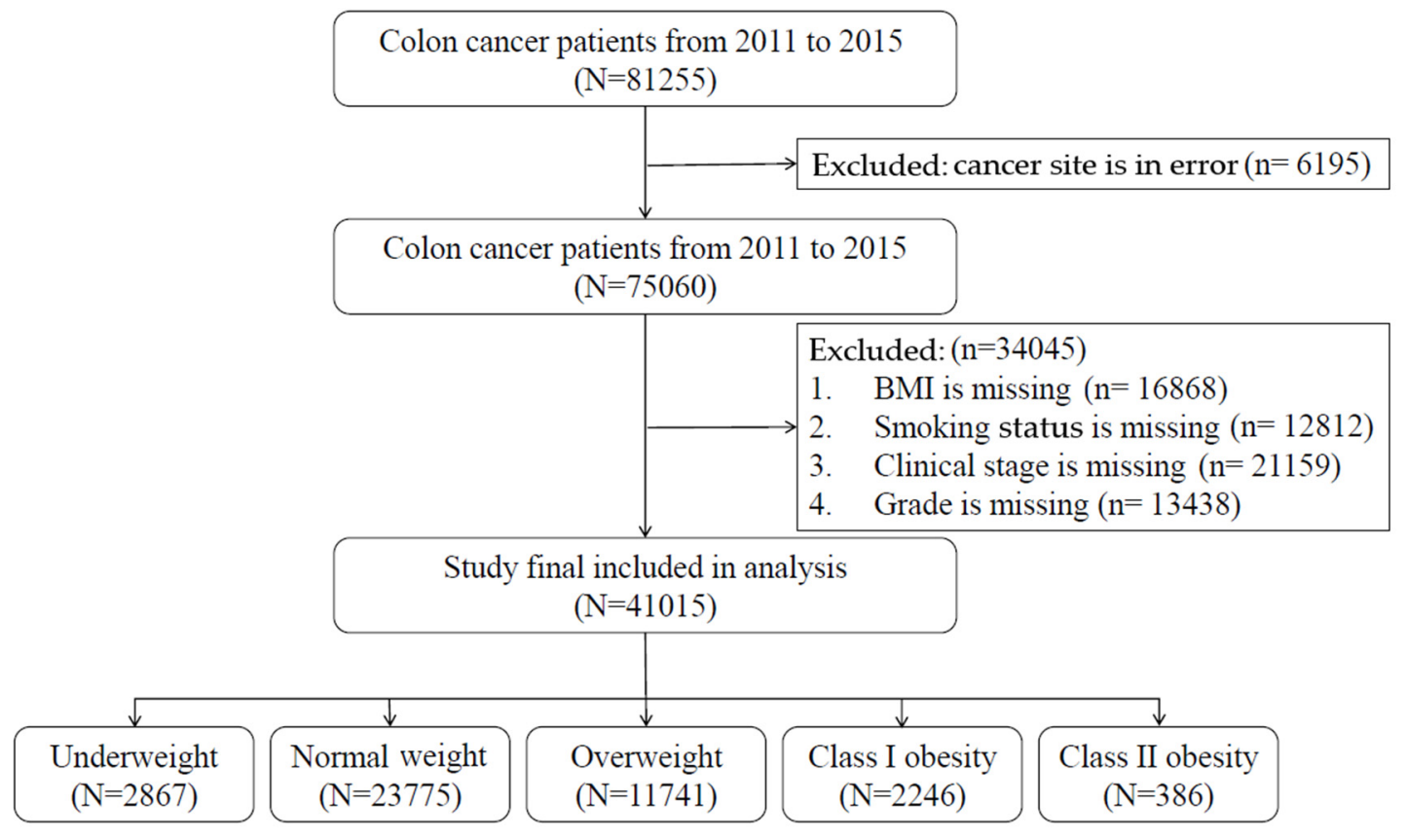

Figure 1. Flowchart of the recruitment and study procedure.

We examined the following survival endpoints: overall survival (OS), defined as the time from pathologic diagnosis to death due to any reason, and disease-free survival (DFS), defined as the time from pathologic diagnosis to disease progression or first recurrence. Besides these, $\mathrm{CRC}$-specific survival was also estimated as the cause of CRC death among those patients. BMI at diagnosis was defined as the BMI at the time of the initial cancer diagnosis. The BMI was categorized into the groups underweight $\left(<18.50 \mathrm{~kg} / \mathrm{m}^{2}\right)$, normal weight $\left(18.50-24.99 \mathrm{~kg} / \mathrm{m}^{2}\right)$, overweight $\left(25.00-29.99 \mathrm{~kg} / \mathrm{m}^{2}\right)$, class I obesity $\left(30.00-34.99 \mathrm{~kg} / \mathrm{m}^{2}\right)$, and class II obesity $\left(\geq 35.00 \mathrm{~kg} / \mathrm{m}^{2}\right)$ according to the 2000 World Health Organization (WHO) guidelines [22].

\subsection{Statistical Analysis}

Summary statistics were presented as the median (range) for continuous variables, and frequency (percentage) for categorical variables. For different BMI groups, continuous variables were analyzed using the Kruskal-Wallis test and categorical variables were analyzed using the Chi-squared test. The trend distributions of the OS, DFS, and CRCspecific survival rates were plotted using the Kaplan-Meier approach with the log-rank test to estimate the statistical differences among the different BMI groups.

The multivariable Cox proportional regression model was used to evaluate hazard ratios (HRs) with 95\% confidence intervals (CIs) of OS, DFS, and CRC-specific survival rates. The HRs of OS, DFS, and CRC-specific survival rates were all presented as age/gender-adjusted HRs, age-/gender-/cancer-stage-adjusted HRs, and adjusted HRs with all selected confounding factors, including age, gender, cancer stage, grade, cancer site, treatment types, smoking status, and comorbidities. A stratified analysis for age group, gender, clinical stage, and cancer site was also conducted. SAS 9.4 (SAS Institute, Inc., Cary, NC, USA) was used for statistical analysis. $p$-values for two-sided analyses were considered statistically significant at $p<0.05$.

\section{Results}

\subsection{Patient Characteristics}

Among our 41,015 CRC patients, there were 57.97\% normal weight, $6.99 \%$ underweight, $28.63 \%$ overweight, $5.47 \%$ class I obesity, and $0.94 \%$ class II obesity patients based 
on the international classification of BMI. Most CRC patients were older than 60 years old (age 60-69, 25.54\%; age $\geq 70,41.42 \%$ ), and $56.64 \%$ were male. Demographic and baseline variables for all CRC patients and the different BMI groups are presented in Table 1. Patients who were underweight presented higher mortality $(56.40 \%)$ and recurrence $(5.34 \%)$ rates.

Table 1. Comparison of the demographic characteristics and underlying comorbidities among different BMI groups with $1 \mathrm{CRC}$

\begin{tabular}{|c|c|c|c|c|c|c|c|}
\hline Characteristic & $\begin{array}{c}\text { Total } \\
(n=41,015)\end{array}$ & $\begin{array}{c}<18.50 \\
(n=2867)\end{array}$ & $\begin{array}{l}18.50-24.99 \\
(n=23,775)\end{array}$ & $\begin{array}{l}25.00-29.99 \\
(n=11,741)\end{array}$ & $\begin{array}{c}30.00-34.99 \\
(n=2246)\end{array}$ & $\begin{array}{c}\geq 35.00 \\
(n=386)\end{array}$ & $p$-Value \\
\hline \multicolumn{8}{|c|}{ Gender, $n(\%)$} \\
\hline Male & $23,230(56.64)$ & $1412(49.25)$ & $13,261(55.78)$ & $7200(61.32)$ & $1191(53.03)$ & $166(43.01)$ & \multirow{2}{*}{$<0.0001$} \\
\hline Female & $17,785(43.36)$ & $1455(50.75)$ & $10,514(44.22)$ & $4541(38.68)$ & 1055 (46.97) & $220(56.99)$ & \\
\hline \multicolumn{8}{|c|}{ Age Group, $n(\%)$} \\
\hline$<40$ & $1349(3.29)$ & $154(5.37)$ & 758 (3.19) & $309(2.63)$ & $98(4.36)$ & $30(7.77)$ & \multirow{5}{*}{$<0.0001$} \\
\hline $40-49$ & $3468(8.46)$ & 209 (7.29) & $2081(8.75)$ & $893(7.61)$ & $236(10.51)$ & 49 (12.69) & \\
\hline $50-59$ & $8737(21.30)$ & $472(16.46)$ & 4909 (20.65) & $2727(23.23)$ & $522(23.24)$ & $107(27.72)$ & \\
\hline $60-69$ & $10,474(25.54)$ & 498 (17.37) & $5816(24.46)$ & 3395 (28.92) & $658(29.30)$ & $107(27.72)$ & \\
\hline$\geq 70$ & $16987(41.42)$ & $1534(53.51)$ & $10,211(42.95)$ & $4417(37.62)$ & 732 (32.59) & $93(24.09)$ & \\
\hline \multicolumn{8}{|c|}{ Clinic Stage, $n(\%)$} \\
\hline 0 & $762(1.82)$ & $40(1.40)$ & $413(1.74)$ & $239(1.89)$ & $54(2.40)$ & $16(4.15)$ & \multirow{5}{*}{$<0.0001$} \\
\hline 1 & $9220(22.00)$ & $448(15.63)$ & 4929 (20.73) & $3069(24.28)$ & 669 (29.79) & $105(27.20)$ & \\
\hline 2 & 8667 (20.68) & $655(22.85)$ & 4561 (19.18) & 2957 (23.39) & $406(18.08)$ & $88(22.80)$ & \\
\hline 3 & $15,203(36.27)$ & $1004(35.02)$ & $8829(37.14)$ & $4468(35.35)$ & $786(35.00)$ & $116(30.05)$ & \\
\hline 4 & 8063 (19.24) & $720(25.11)$ & $5043(21.21)$ & 1908 (15.09) & 331 (14.74) & $61(15.80)$ & \\
\hline \multicolumn{8}{|c|}{ Site, $n(\%)$} \\
\hline $\begin{array}{l}\text { Malignant } \\
\text { neoplasm of } \\
\text { colon }\end{array}$ & $26,153(63.76)$ & 1847 (64.42) & $15,293(64.32)$ & 7301 (62.18) & 1448 (64.47) & $264(68.39)$ & \multirow{3}{*}{0.0073} \\
\hline $\begin{array}{l}\text { Rectosigmoid } \\
\text { junction }\end{array}$ & 3441 (8.39) & $234(8.16)$ & $1975(8.31)$ & 1015 (8.64) & $188(8.37)$ & $29(7.51)$ & \\
\hline Rectum & $11,421(27.85)$ & 786 (27.42) & 6507 (27.37) & 3425 (29.17) & $610(27.16)$ & $93(24.09)$ & \\
\hline \multicolumn{8}{|c|}{ Treatment, $n$ (\%) } \\
\hline Operation & $37,240(90.80)$ & $2401(83.75)$ & $21,486(90.37)$ & $10,886(92.72)$ & 2111 (93.99) & $356(92.23)$ & $<0.0001$ \\
\hline Radiotherapy & $4782(11.66)$ & $323(11.27)$ & 2827 (11.89) & 1366 (11.63) & $221(9.84)$ & 45 (11.66) & 0.0638 \\
\hline Chemotherapy & $24,760(60.37)$ & $1572(54.83)$ & $14,618(61.48)$ & $7063(60.16)$ & $1292(57.52)$ & $215(55.70)$ & $<0.0001$ \\
\hline \multicolumn{8}{|c|}{ Smoking, $n(\%)$} \\
\hline Never & $29,872(72.83)$ & $2070(72.20)$ & $17,428(73.30)$ & $8396(71.51)$ & $1675(74.58)$ & $303(78.50)$ & \multirow{3}{*}{$<0.0001$} \\
\hline Quit & 4415 (10.76) & $280(9.77)$ & 2447 (10.29) & $1410(12.01)$ & 249 (11.09) & $29(7.51)$ & \\
\hline Current & $6728(16.40)$ & 517 (18.03) & $3900(16.40)$ & 1935 (16.48) & 322 (14.34) & 54 (13.99) & \\
\hline Death, $n(\%)$ & $15,021(36.62)$ & $1617(56.40)$ & 9097 (38.26) & $3550(30.24)$ & 655 (29.16) & 102 (26.42) & $<0.0001$ \\
\hline $\begin{array}{l}\text { Time to mortality, } \\
\text { Median (Q1-Q3) }\end{array}$ & $1.49(0.64-2.62)$ & $0.97(0.37-2.01)$ & $1.48(0.64-2.58)$ & $1.71(0.79-2.88)$ & $1.82(0.88-2.96)$ & $1.69(0.79-2.92)$ & $<0.0001$ \\
\hline $\begin{array}{l}\text { Death in colon } \\
\text { cancer, } n(\%)\end{array}$ & 11,347 (27.67) & 1186 (41.37) & $6911(29.07)$ & $2696(22.96)$ & $483(21.50)$ & $71(18.39)$ & $<0.0001$ \\
\hline $\begin{array}{l}\text { Time to specific- } \\
\text { mortality, } \\
\text { Median (Q1-Q3) }\end{array}$ & $1.41(0.61-2.43)$ & $0.93(0.33-1.88)$ & $1.38(0.60-2.38)$ & $1.62(0.77-2.65)$ & $1.77(0.88-2.86)$ & $1.53(0.76-2.83)$ & $<0.0001$ \\
\hline Recurrence, $n(\%)$ & $2068(5.04)$ & $153(5.34)$ & $1218(5.12)$ & $576(4.91)$ & $106(4.72)$ & $15(3.89)$ & 0.0016 \\
\hline $\begin{array}{c}\text { Time to } \\
\text { recurrence, } \\
\text { Median (Q1-Q3) }\end{array}$ & $0.64(0.44-0.84)$ & $0.56(0.41-0.78)$ & $0.64(0.44-0.83)$ & $0.65(0.45-0.85)$ & $0.63(0.46-0.85)$ & $0.69(0.41-1.00)$ & 0.0402 \\
\hline
\end{tabular}


Table 1. Cont.

\begin{tabular}{|c|c|c|c|c|c|c|c|}
\hline Characteristic & $\begin{array}{c}\text { Total } \\
(n=41,015)\end{array}$ & $\begin{array}{c}<18.50 \\
(n=2867)\end{array}$ & $\begin{array}{l}18.50-24.99 \\
(n=23,775)\end{array}$ & $\begin{array}{l}25.00-29.99 \\
(n=11,741)\end{array}$ & $\begin{array}{c}30.00-34.99 \\
(n=2246)\end{array}$ & $\begin{array}{c}\geq 35.00 \\
(n=386)\end{array}$ & $p$-Value \\
\hline \multicolumn{8}{|c|}{ Comorbidity, $n$ (\%) } \\
\hline $\begin{array}{l}\text { Myocardial } \\
\text { infarction }\end{array}$ & $482(1.18)$ & $41(1.43)$ & $238(1.00)$ & $164(1.40)$ & $35(1.56)$ & $4(1.01)$ & 0.0035 \\
\hline $\begin{array}{l}\text { Congestive } \\
\text { heart failure }\end{array}$ & $1977(4.82)$ & $152(5.30)$ & 1044 (4.39) & $588(5.01)$ & $167(7.44)$ & $26(6.74)$ & $<0.0001$ \\
\hline $\begin{array}{l}\text { Peripheral } \\
\text { vascular } \\
\text { disease }\end{array}$ & $752(1.83)$ & $51(1.78)$ & $433(1.82)$ & $219(1.87)$ & $41(1.83)$ & $8(2.07)$ & 0.9924 \\
\hline $\begin{array}{c}\text { Cardiovascular } \\
\text { disease }\end{array}$ & $3396(8.28)$ & $289(10.08)$ & 1975 (8.31) & $930(7.92)$ & $184(8.19)$ & $18(4.66)$ & 0.0003 \\
\hline Dementia & $921(2.25)$ & $161(5.62)$ & 569 (2.39) & $170(1.45)$ & $20(0.89)$ & $1(0.26)$ & $<0.0001$ \\
\hline COPD & 3339 (8.14) & 307 (10.71) & $1816(7.64)$ & $951(8.10)$ & $213(9.48)$ & $52(13.47)$ & $<0.0001$ \\
\hline $\begin{array}{l}\text { Rheumatic } \\
\text { disease }\end{array}$ & $432(1.05)$ & 33 (1.15) & $261(1.10)$ & $121(1.03)$ & $13(0.58)$ & $4(1.04)$ & 0.2289 \\
\hline $\begin{array}{l}\text { peptic ulcer } \\
\text { disease }\end{array}$ & $5966(14.55)$ & 514 (17.93) & 3462 (14.56) & $1625(13.84)$ & $297(13.22)$ & 68 (17.62) & $<0.0001$ \\
\hline $\begin{array}{l}\text { Mild liver } \\
\text { disease }\end{array}$ & 3151 (7.68) & $196(6.84)$ & $1702(7.16)$ & $1006(8.57)$ & $212(9.44)$ & $35(9.07)$ & $<0.0001$ \\
\hline $\begin{array}{c}\text { Diabetes } \\
\text { without } \\
\text { chronic } \\
\text { complication }\end{array}$ & 8805 (21.47) & $362(12.63)$ & 4630 (19.47) & $2992(25.48)$ & $682(30.37)$ & $139(36.01)$ & $<0.0001$ \\
\hline $\begin{array}{l}\text { Diabetes with } \\
\text { chronic } \\
\text { complication }\end{array}$ & $2180(5.32)$ & $77(2.69)$ & $1123(4.72)$ & $762(6.49)$ & $184(8.19)$ & $34(8.81)$ & $<0.0001$ \\
\hline $\begin{array}{c}\text { Hemiplegia or } \\
\text { paraplegia }\end{array}$ & $287(0.70)$ & $27(0.94)$ & $175(0.74)$ & $70(0.60)$ & $12(0.53)$ & $3(0.78)$ & 0.2313 \\
\hline Renal disease & $2188(5.33)$ & $144(5.02)$ & $1244(5.23)$ & $635(5.41)$ & $146(6.50)$ & $19(4.92)$ & 0.1187 \\
\hline $\begin{array}{l}\text { Moderate or } \\
\text { severe liver } \\
\text { disease }\end{array}$ & $157(0.38)$ & $8(0.28)$ & $89(0.37)$ & $48(0.41)$ & $11(0.49)$ & $1(0.26)$ & 0.7557 \\
\hline \multicolumn{8}{|c|}{ Grade, $n(\%)$} \\
\hline $\begin{array}{c}\text { 1. Well } \\
\text { differentiated }\end{array}$ & 3266 (7.96) & $191(6.66)$ & 1859 (7.96) & $974(8.30)$ & $202(8.99)$ & 40 (10.36) & \multirow{5}{*}{$<0.0001$} \\
\hline $\begin{array}{l}\text { 2. Moderately } \\
\text { differentiated }\end{array}$ & $33,134(80.79)$ & $2312(80.64)$ & $19,139(80.38)$ & 9571 (81.52) & $1811(80.63)$ & 301 (77.98) & \\
\hline $\begin{array}{c}\text { 3. Poorly } \\
\text { differentiated }\end{array}$ & 3734 (9.10) & $285(9.94)$ & 2268 (9.53) & $969(8.25)$ & $176(7.84)$ & $36(9.33)$ & \\
\hline $\begin{array}{l}\text { 4. Undifferenti- } \\
\text { ated }\end{array}$ & $393(0.96)$ & 33 (1.15) & $234(0.98)$ & $103(0.88)$ & $20(0.89)$ & $3(0.78)$ & \\
\hline 5. Others & 488 (1.19) & $46(1.60)$ & $275(1.15)$ & $124(1.06)$ & 37 (1.65) & $6(1.55)$ & \\
\hline
\end{tabular}

p.s. The time of median follow up was 3.27 (2.08-4.82) years.

\subsection{Hazard Ratios of BMI at Diagnosis and Mortality Rates}

Table 2 shows that patients belonging to the underweight group had worse OS (aHR: 1.61; 95\% CI: 1.53-1.70; $p$-value: <0.0001) and CRC-specific survival (aHR: 1.52; 95\% CI: 1.43-1.62; $p$-value: $<0.0001$ ) rates than those patients of normal weight after adjusting for selected confounding factors. However, patients in the overweight, class I obesity, and 
class II obesity groups showed a statistically significant protective effect on OS and CRCspecific survival rates compared with those of normal weight. We also found a statistically significant risk of underweight BMI on DFS rates when compared to CRC patients with a normal weight.

Table 2. Hazard Ratios of BMI at Diagnosis and Mortality Rates.

\begin{tabular}{|c|c|c|c|c|c|c|c|c|}
\hline Characteristic & $\begin{array}{c}\text { Patient } \\
(n=41015)\end{array}$ & $\begin{array}{c}\text { Event } \\
(n=15021)\end{array}$ & $\begin{array}{l}\text { Age-, Gender- } \\
\text { Adjusted HR }\end{array}$ & $p$-Value & $\begin{array}{c}\text { Age-, Gender-, Stage- } \\
\text { Adjusted HR }\end{array}$ & $p$-Value & $\begin{array}{l}\text { Multivariable- } \\
\text { Adjusted HR }{ }^{\dagger}\end{array}$ & $p$-Value \\
\hline \multicolumn{9}{|c|}{ Overall Survival } \\
\hline \multicolumn{9}{|c|}{ BMI WHO Categories, $n(\%)$} \\
\hline$<18.50$ & 2867 & $1617(56.40)$ & $1.76(1.67-1.85)$ & \multirow{2}{*}{$<0.0001$} & $1.68(1.59-1.77)$ & \multirow{2}{*}{$<0.0001$} & $1.61(1.53-1.70)$ & \multirow{2}{*}{$<0.0001$} \\
\hline $18.50-24.99$ & 23,775 & $9097(38.26)$ & Ref. & & Ref. & & Ref. & \\
\hline $25.00-29.99$ & 11,741 & $3550(30.24)$ & $0.75(0.72-0.78)$ & $<0.0001$ & $0.82(0.79-0.86)$ & $<0.0001$ & $0.82(0.79-0.85)$ & $<0.0001$ \\
\hline $30.00-34.99$ & 2246 & $655(29.16)$ & $0.76(0.71-0.83)$ & $<0.0001$ & $0.87(0.80-0.94)$ & $<0.0001$ & $0.83(0.77-0.90)$ & $<0.0001$ \\
\hline$\geq 35.00$ & 386 & $102(26.42)$ & $0.73(0.60-0.89)$ & 0.0018 & $0.83(0.68-1.01)$ & $<0.0001$ & $0.74(0.61-0.90)$ & 0.0027 \\
\hline \multicolumn{9}{|c|}{ CRC-Specific Survival } \\
\hline \multicolumn{9}{|c|}{ BMI WHO Categories, $\boldsymbol{n}(\%)$} \\
\hline$<18.50$ & 2867 & $1186(41.37)$ & $1.69(1.59-1.79)$ & \multirow{2}{*}{$<0.0001$} & $1.59(1.50-1.69)$ & \multirow{2}{*}{$<0.0001$} & $1.52(1.43-1.62)$ & \multirow{2}{*}{$<0.0001$} \\
\hline $18.50-24.99$ & 23,775 & $6911(29.07)$ & Ref. & & Ref. & & Ref. & \\
\hline $25.00-29.99$ & 11,741 & $2696(22.96)$ & $0.75(0.72-0.79)$ & $<0.0001$ & $0.85(0.81-0.88)$ & $<0.0001$ & $0.85(0.81-0.89)$ & $<0.0001$ \\
\hline $30.00-34.99$ & 2246 & $483(21.50)$ & $0.73(0.66-0.80)$ & $<0.0001$ & $0.86(0.79-0.95)$ & 0.0015 & $0.85(0.78-0.94)$ & 0.0009 \\
\hline$\geq 35.00$ & 386 & $71(18.39)$ & $0.64(0.50-0.80)$ & 0.0001 & $0.74(0.59-0.94)$ & 0.0119 & $0.69(0.54-0.87)$ & 0.0017 \\
\hline \multicolumn{9}{|c|}{ Disease Free Survival $^{\#}$} \\
\hline \multicolumn{9}{|c|}{ BMI WHO categories, $n(\%)$} \\
\hline$<18.50$ & 1853 & $737(39.77)$ & $1.73(1.60-1.87)$ & \multirow{2}{*}{$<0.0001$} & $1.71(1.58-1.85)$ & \multirow{2}{*}{$<0.0001$} & $1.75(1.62-1.89)$ & \multirow{2}{*}{$<0.0001$} \\
\hline $18.50-24.99$ & 17,660 & $4454(25.22)$ & Ref. & & Ref. & & Ref. & \\
\hline $25.00-29.99$ & 9417 & $1932(20.52)$ & $0.81(0.77-0.85)$ & $<0.0001$ & $0.83(0.78-0.87)$ & $<0.0001$ & $0.81(0.77-0.86)$ & $<0.0001$ \\
\hline $30.00-34.99$ & 1825 & $381(20.88)$ & $0.89(0.80-0.99)$ & 0.0329 & $0.94(0.84-1.04)$ & 0.2212 & $0.87(0.79-0.97)$ & 0.0122 \\
\hline$\geq 35.00$ & 308 & $53(17.21)$ & $0.79(0.60-1.03)$ & 0.0797 & $0.83(0.64-1.09)$ & 0.1852 & $0.75(0.57-0.98)$ & 0.0351 \\
\hline
\end{tabular}

${ }^{\dagger}$ Multivariable-adjusted HRs were adjusted for age, gender, stage, grade, cancer site, treatment types, smoking status, and comorbidities.

\# Excluding 9952 patients whose cancer recurrence were unknown.

\subsection{Stratified Analyses of Overall Survival Rates}

The stratified OS rates for age, gender, clinical stage, and cancer site are presented in Table 3. In patients aged $<50$ years, there were no significant differences between the normal weight group and the other four BMI groups. However, in those older than 50 , a significantly lower OS rate was noted in the underweight group compared to the normal weight group. On the contrary, a significantly better OS rate was noted in the overweight group older than 50 and in the class I and II obesity groups older than 70 compared to that in the normal weight group. A worse OS rate was noted in underweight patients of both genders. A statistically significantly better OS rate was noted in patients from the overweight, class I obesity, and class II obesity groups, except in class II obese males. Considering the clinical stage factor, we also found the same adverse results for underweight patients of all stages. Nevertheless, we noted that the OS rate for stage 0 was lower than that for other stages, which might be related to the bias caused by a smaller patient number in that group. A worse OS rate was also noted in patients in the all-stage/overweight groups, stage 2/class I obese group, and stage 4/class II obese group. 
Table 3. Stratified analyses of overall survival rates.

\begin{tabular}{|c|c|c|c|c|c|}
\hline \multirow{2}{*}{ Characteristic } & \multicolumn{5}{|c|}{ BMI WHO Categories } \\
\hline & $<18.50$ & $18.50-24.99$ & 25.00-29.99 & $30.00-34.99$ & $\geq 35.00$ \\
\hline \multicolumn{6}{|c|}{ Age Group } \\
\hline$<40, \mathrm{No}$ & 154 & 758 & 309 & 98 & 30 \\
\hline Event, No & 51 & 251 & 85 & 34 & 9 \\
\hline $\mathrm{HR}(95 \% \mathrm{CI})$ & $0.96(0.70-1.31)$ & Ref. & $0.96(0.74-1.24)$ & $1.14(0.79-1.65)$ & $0.69(0.35-1.37)$ \\
\hline $40-49, \mathrm{No}$ & 209 & 2081 & 893 & 236 & 49 \\
\hline Event, No & 86 & 595 & 238 & 48 & 9 \\
\hline HR (95\% CI) & $1.23(0.98-1.54)$ & Ref. & $1.03(0.88-1.20)$ & $0.81(0.60-1.09)$ & $0.77(0.40-1.50)$ \\
\hline $50-59, \mathrm{No}$ & 472 & 4909 & 2727 & 522 & 107 \\
\hline Event, No & 194 & 1407 & 638 & 127 & 25 \\
\hline $\operatorname{HR}(95 \% \mathrm{CI})$ & $1.49(1.28-1.73)^{* *}$ & Ref. & $0.84(0.76-0.92) *$ & $1.01(0.84-1.22)$ & $0.76(0.51-1.14)$ \\
\hline $60-69, \mathrm{No}$ & 498 & 5816 & 3395 & 658 & 107 \\
\hline Event, No & 237 & 1728 & 821 & 149 & 20 \\
\hline $\operatorname{HR}(95 \% \mathrm{CI})$ & $1.85(1.61-2.13) * *$ & Ref. & $0.90(0.83-0.98) *$ & $0.88(0.75-1.05)$ & $0.85(0.55-1.32)$ \\
\hline$\geq 70$, No & 1534 & 10,211 & 4417 & 732 & 93 \\
\hline Event, No & 1049 & 5116 & 1768 & 297 & 39 \\
\hline $\operatorname{HR}(95 \% \mathrm{CI})$ & $1.62(1.51-1.73)^{* *}$ & Ref. & $0.77(0.73-0.81)^{* *}$ & $0.77(0.69-0.87) * *$ & $0.75(0.54-1.02)$ \\
\hline \multicolumn{6}{|c|}{ Gender } \\
\hline Male, No & 1412 & 13,261 & 7200 & 1191 & 166 \\
\hline Event, No & 888 & 5436 & 2210 & 361 & 46 \\
\hline $\operatorname{HR}(95 \% \mathrm{CI})$ & $1.72(1.60-1.85)^{* *}$ & Ref. & $0.79(0.75-0.83)^{* *}$ & $0.84(0.76-0.94) *$ & $0.79(0.59-1.06)$ \\
\hline Female, No & 1455 & 10,514 & 4541 & 1055 & 220 \\
\hline Event, No & 729 & 3661 & 1340 & 294 & 56 \\
\hline $\operatorname{HR}(95 \% \mathrm{CI})$ & $1.48(1.36-1.60) * *$ & Ref. & $0.85(0.80-0.91)^{* *}$ & $0.81(0.72-0.92) *$ & $0.69(0.53-0.90)$ * \\
\hline \multicolumn{6}{|c|}{ Clinic Stage } \\
\hline Stage 0, No & 40 & 413 & 239 & 54 & 16 \\
\hline Event, No & 18 & 57 & 15 & 3 & 1 \\
\hline $\operatorname{HR}(95 \% \mathrm{CI})$ & $3.72(1.95-7.11) * *$ & Ref. & $0.45(0.25-0.82) *$ & $0.32(0.09-1.09)$ & $0.43(0.05-3.53)$ \\
\hline Stage 1, No & 448 & 4929 & 3069 & 669 & 105 \\
\hline Event, No & 171 & 937 & 412 & 102 & 13 \\
\hline $\operatorname{HR}(95 \% \mathrm{CI})$ & $2.23(1.88-2.64) * *$ & Ref. & $0.69(0.61-0.78) * *$ & $0.85(0.69-1.04)$ & $0.72(0.41-1.24)$ \\
\hline Stage $2, \mathrm{No}$ & 655 & 4561 & 2957 & 406 & 88 \\
\hline Event, No & 321 & 1340 & 483 & 87 & 18 \\
\hline $\operatorname{HR}(95 \% \mathrm{CI})$ & $1.94(1.71-2.19)^{* *}$ & Ref. & $0.74(0.67-0.82) * *$ & $0.72(0.58-0.89) *$ & $0.75(0.47-1.21)$ \\
\hline Stage 3, No & 1004 & 8829 & 4468 & 786 & 116 \\
\hline Event, No & 487 & 2699 & 1144 & 206 & 29 \\
\hline $\operatorname{HR}(95 \% \mathrm{CI})$ & $1.85(1.68-2.04)^{* *}$ & Ref. & $0.82(0.77-0.88) * *$ & $0.88(0.76-1.01)$ & $0.90(0.62-1.30)$ \\
\hline Stage 4 , No & 720 & 5043 & 1908 & 331 & 61 \\
\hline Event, No & 620 & 4064 & 1496 & 257 & 41 \\
\hline $\operatorname{HR}(95 \% \mathrm{CI})$ & $1.23(1.13-1.34)^{* *}$ & Ref. & $0.90(0.85-0.96) *$ & $0.90(0.79-1.02)$ & $0.68(0.50-0.93)$ * \\
\hline \multicolumn{6}{|c|}{ Site } \\
\hline $\begin{array}{l}\text { Malignant neoplasm } \\
\text { of colon, No }\end{array}$ & 1847 & 15,293 & 7301 & 1448 & 264 \\
\hline Event, No & 1018 & 5853 & 2215 & 428 & 72 \\
\hline $\operatorname{HR}(95 \% \mathrm{CI})$ & $1.56(1.46-1.67) * *$ & Ref. & $0.82(0.78-0.86)^{* *}$ & $0.84(0.76-0.93) *$ & $0.84(0.67-1.06)$ \\
\hline $\begin{array}{l}\text { Rectosigmoid } \\
\text { junction, No }\end{array}$ & 234 & 1975 & 1015 & 188 & 29 \\
\hline Event, No & 137 & 757 & 294 & 53 & 5 \\
\hline $\operatorname{HR}(95 \% \mathrm{CI})$ & $1.66(1.38-2.01)^{* *}$ & Ref. & $0.81(0.71-0.93) *$ & $0.79(0.60-1.05)$ & $0.32(0.13-0.78)$ * \\
\hline Rectum, No & 786 & 6507 & 3425 & 610 & 93 \\
\hline Event, No & 462 & 2487 & 1041 & 174 & 25 \\
\hline $\operatorname{HR}(95 \% \mathrm{CI})$ & $1.78(1.61-1.97) * *$ & Ref. & $0.82(0.76-0.88)^{* *}$ & $0.83(0.71-0.97)$ * & $0.70(0.47-1.03)$ \\
\hline
\end{tabular}

${ }^{*} p<0.05,{ }^{* *} p<0.0001$.

\subsection{Stratified Analyses of Disease-Free Survival Rates}

Table 4 presents the stratified DFS rates. In patients aged $<50$ years, there was no significant difference between the normal weight group and the other four BMI category 
groups. However, a significantly worse DFS rate was noted in the underweight group than in the normal weight group, which was mainly present in the subgroup older than 50. On the contrary, a significantly better DFS rate was noted in the overweight group older than 50 and in the class I obesity group older than 70, a similar trend to the stratified OS rate in Table 3. A worse DFS rate was noted in underweight patients of both genders. A statistically significantly better DFS rate was noted in male patients in the overweight and class I obese groups. As for the clinical stage factor, adverse results were noted in the underweight patients in stages $0,1,2$, and 3 . Lower HRs were also noted in overweight patients in stages $0,1,2$, and 3 and in class I obese patients in stages 0 and 2.

Table 4. Stratified analyses of disease-free survival rates.

\begin{tabular}{|c|c|c|c|c|c|}
\hline \multirow{2}{*}{ Characteristic } & \multicolumn{5}{|c|}{ WHO Categories } \\
\hline & $<18.50$ & 18.50-24.99 & $25.00-29.99$ & $30.00-34.99$ & $\geq 35.00$ \\
\hline \multicolumn{6}{|c|}{ Age Group } \\
\hline$<40, \mathrm{No}$ & 104 & 528 & 233 & 73 & 24 \\
\hline Event, No & 18 & 84 & 38 & 16 & 4 \\
\hline HR (95\% CI) & $1.06(0.63-1.79)$ & Ref. & $1.05(0.71-1.57)$ & $1.41(0.81-2.44)$ & $0.86(0.30-2.42)$ \\
\hline $40-49$, No & 142 & 1561 & 696 & 193 & 37 \\
\hline Event, No & 33 & 232 & 105 & 28 & 2 \\
\hline $\mathrm{HR}(95 \% \mathrm{CI})$ & $1.30(0.89-1.90)$ & Ref. & $1.07(0.84-1.36)$ & $0.95(0.64-1.42)$ & $0.35(0.09-1.42)$ \\
\hline $50-59$, No & 337 & 3760 & 2221 & 436 & 86 \\
\hline Event, No & 83 & 629 & 325 & 75 & 14 \\
\hline HR $(95 \%$ CI $)$ & $1.50(1.19-1.90)$ * & Ref. & $0.88(0.77-1.00) *$ & $1.05(0.82-1.34)$ & $1.05(0.61-1.80)$ \\
\hline $60-69$, No & 330 & 4498 & 2842 & 554 & 89 \\
\hline Event, No & 95 & 796 & 446 & 88 & 11 \\
\hline HR (95\% CI) & $1.92(1.55-2.38) * *$ & Ref. & $0.88(0.79-0.99)$ * & $0.92(0.74-1.15)$ & $0.76(0.42-1.37)$ \\
\hline$\geq 70$, No & 940 & 7313 & 3425 & 569 & 72 \\
\hline Event, No & 508 & 2713 & 1018 & 174 & 22 \\
\hline HR $(95 \%$ CI) & $1.79(1.62-1.97) * *$ & Ref. & $0.75(0.70-0.81)^{* *}$ & $0.79(0.67-0.92)$ * & $0.73(0.48-1.11)$ \\
\hline \multicolumn{6}{|c|}{ Gender } \\
\hline Male, No & 878 & 9721 & 5728 & 970 & 139 \\
\hline Event, No & 423 & 2725 & 1165 & 209 & 29 \\
\hline HR $(95 \%$ CI $)$ & $1.88(1.70-2.09) * *$ & Ref. & $0.75(0.70-0.80)^{* *}$ & $0.84(0.73-0.97) *$ & $0.82(0.57-1.19)$ \\
\hline Female, No & 975 & 7939 & 3689 & 855 & 169 \\
\hline Event, No & 314 & 1729 & 767 & 172 & 24 \\
\hline $\mathrm{HR}(95 \% \mathrm{CI})$ & $1.59(1.41-1.80)^{* *}$ & Ref. & $0.92(0.85-1.00) *$ & $0.93(0.79-1.08)$ & $0.71(0.47-1.06)$ \\
\hline \multicolumn{6}{|c|}{ Clinic Stage } \\
\hline Stage 0, No & 34 & 385 & 223 & 49 & 15 \\
\hline Event, No & 12 & 49 & 14 & 2 & 1 \\
\hline HR $(95 \%$ CI $)$ & $3.24(1.52-6.90)$ * & Ref. & $0.41(0.22-0.77) *$ & $0.20(0.04-0.93)$ * & $0.27(0.03-2.35)$ \\
\hline Stage 1, No & 388 & 4619 & 2906 & 641 & 96 \\
\hline Event, No & 128 & 818 & 365 & 100 & 9 \\
\hline $\operatorname{HR}(95 \% \mathrm{CI})$ & $2.06(1.70-2.49) * *$ & Ref. & $0.69(0.61-0.78)^{* *}$ & $0.92(0.74-1.13)$ & $0.56(0.29-1.07)$ \\
\hline Stage 2, No & 540 & 4065 & 1880 & 371 & 80 \\
\hline Event, No & 232 & 1082 & 417 & 76 & 18 \\
\hline HR (95\% CI) & $1.85(1.61-2.14)^{* *}$ & Ref. & $0.78(0.70-0.88)^{* *}$ & $0.75(0.59-0.94)$ * & $0.95(0.60-1.52)$ \\
\hline Stage 3, No & 798 & 7780 & 4055 & 703 & 101 \\
\hline Event, No & 319 & 2130 & 988 & 177 & 20 \\
\hline HR $(95 \%$ CI $)$ & $1.64(1.46-1.85)^{* *}$ & Ref. & $0.88(0.81-0.95) *$ & $0.94(0.81-1.10)$ & $0.77(0.50-1.20)$ \\
\hline Stage 4, No & 93 & 811 & 353 & 61 & 16 \\
\hline Event, No & 46 & 375 & 148 & 26 & 5 \\
\hline HR (95\% CI) & $1.23(0.90-1.68)$ & Ref. & $0.89(0.73-1.08)$ & $0.85(0.57-1.27)$ & $0.68(0.28-1.69)$ \\
\hline
\end{tabular}


Table 4. Cont.

\begin{tabular}{|c|c|c|c|c|c|}
\hline \multirow{2}{*}{ Characteristic } & \multicolumn{5}{|c|}{ WHO Categories } \\
\hline & $<18.50$ & $18.50-24.99$ & $25.00-29.99$ & $30.00-34.99$ & $\geq 35.00$ \\
\hline \multicolumn{6}{|c|}{ Site } \\
\hline $\begin{array}{l}\text { Malignant neoplasm } \\
\text { of colon, No }\end{array}$ & 1248 & 11397 & 5850 & 1169 & 212 \\
\hline Event, No & 492 & 2856 & 1156 & 233 & 39 \\
\hline $\operatorname{HR}(95 \% \mathrm{CI})$ & $1.69(1.54-1.87) * *$ & Ref. & $0.78(0.73-0.84)^{* *}$ & $0.83(0.73-0.96)$ * & $0.89(0.65-1.23)$ \\
\hline $\begin{array}{l}\text { Rectosigmoid } \\
\text { junction, No }\end{array}$ & 151 & 1480 & 851 & 147 & 26 \\
\hline Event, No & 67 & 372 & 192 & 28 & 3 \\
\hline $\operatorname{HR}(95 \%$ CI) & $1.99(1.53-2.60)^{* *}$ & Ref. & $0.91(0.76-1.09)$ & $0.81(0.55-1.19)$ & $0.21(0.06-0.67)$ * \\
\hline Rectum, No & 454 & 4783 & 2716 & 509 & 70 \\
\hline Event, No & 178 & 1226 & 584 & 120 & 11 \\
\hline $\operatorname{HR}(95 \%$ CI) & $1.83(1.56-2.15)^{* *}$ & Ref. & $0.84(0.76-0.93) *$ & $1.00(0.83-1.20)$ & $0.65(0.36-1.18)$ \\
\hline
\end{tabular}

\subsection{Stratified Analyses of Colorectal-Cancer-Specific Survival Rates}

Table 5 presents the stratified CRC-specific survival estimates. In patients aged $<50$ years, there was no significant difference between the normal weight group and the other four BMI category groups. However, in those older than 50, the CRC-specific survival rate in the underweight group was significantly lower. Besides this, the CRC-specific survival rate was higher in overweight patients aged $50-59$ or $\geq 70$ years and in class I obese patients aged $\geq 70$ years. For the gender factor, we noted a statistically significantly worse CRC-specific survival rate in the underweight patients and better CRC-specific survival rates in the overweight, class I obese, and class II obese patient groups. In relation to the clinical stage, adverse results were noted in all underweight patients. On the contrary, a protective effect was noted in patients in stages 1, 2, 3, and 4, class I obese patients in stage 2 , and class II obese patients in stages 2 and 4 .

Table 5. Stratified analyses of CRC-specific survival rates.

\begin{tabular}{|c|c|c|c|c|c|}
\hline \multirow{2}{*}{ Characteristic } & \multicolumn{5}{|c|}{ WHO Categories } \\
\hline & $<18.50$ & $18.50-24.99$ & $25.00-29.99$ & $30.00-34.99$ & $\geq 35.00$ \\
\hline \multicolumn{6}{|c|}{ Age Group } \\
\hline$<40$, No & 154 & 758 & 309 & 98 & 30 \\
\hline Event, No & 49 & 236 & 79 & 31 & 9 \\
\hline HR $(95 \%$ CI) & $1.00(0.73-1.38)$ & Ref. & $0.94(0.72-1.23)$ & $1.11(0.75-1.64)$ & $0.76(0.38-1.50)$ \\
\hline $40-49, \mathrm{No}$ & 209 & 2081 & 893 & 236 & 49 \\
\hline Event, No & 78 & 543 & 218 & 41 & 6 \\
\hline HR $(95 \%$ CI) & $1.24(0.97-1.57)$ & Ref. & $1.06(0.90-1.24)$ & $0.82(0.59-1.13)$ & $0.57(0.25-1.27)$ \\
\hline $50-59$, No & 472 & 4909 & 2727 & 522 & 107 \\
\hline Event, No & 158 & 1214 & 547 & 101 & 19 \\
\hline HR $(95 \%$ CI $)$ & $1.38(1.17-1.64) *$ & Ref. & $0.87(0.78-0.96) *$ & $1.04(0.85-1.28)$ & $0.68(0.43-1.08)$ \\
\hline $60-69, \mathrm{No}$ & 498 & 5816 & 3395 & 658 & 107 \\
\hline Event, No & 183 & 1370 & 644 & 117 & 12 \\
\hline HR $(95 \%$ CI) & $1.76(1.51-2.06)^{* *}$ & Ref. & $0.94(0.86-1.04)$ & $0.96(0.79-1.16)$ & $0.73(0.41-1.29)$ \\
\hline$\geq 70$, No & 1534 & 10,211 & 4417 & 732 & 93 \\
\hline Event, No & 718 & 3548 & 1208 & 193 & 25 \\
\hline $\operatorname{HR}(95 \% \mathrm{CI})$ & $1.54(1.42-1.67) * *$ & Ref. & $0.79(0.74-0.84)^{* *}$ & $0.76(0.66-0.88)$ * & $0.74(0.50-1.10)$ \\
\hline
\end{tabular}


Table 5. Cont.

\begin{tabular}{|c|c|c|c|c|c|}
\hline \multirow{2}{*}{ Characteristic } & \multicolumn{5}{|c|}{ WHO Categories } \\
\hline & $<18.50$ & $18.50-24.99$ & $25.00-29.99$ & $30.00-34.99$ & $\geq 35.00$ \\
\hline \multicolumn{6}{|c|}{ Gender } \\
\hline Male, No & 1412 & 13261 & 7200 & 1191 & 166 \\
\hline Event, No & 616 & 3973 & 1678 & 252 & 28 \\
\hline HR $(95 \%$ CI $)$ & $1.62(1.49-1.77)^{* *}$ & Ref. & $0.84(0.80-0.89)^{* *}$ & $0.85(0.75-0.97)$ * & $0.69(0.47-1.00)$ * \\
\hline Female, No & 1455 & 10,514 & 4541 & 1055 & 220 \\
\hline Event, No & 570 & 2938 & 1018 & 231 & 43 \\
\hline HR $(95 \%$ CI) & $1.43(1.30-1.56)^{* *}$ & Ref. & $0.85(0.79-0.92)^{* *}$ & $0.84(0.74-0.96)$ * & $0.67(0.49-0.90)$ * \\
\hline \multicolumn{6}{|c|}{ Clinic Stage } \\
\hline Stage 0, No & 40 & 413 & 239 & 54 & 16 \\
\hline Event, No & 4 & 11 & 2 & 1 & 1 \\
\hline HR (95\% CI) & $9.29(1.42-60.60) *$ & Ref. & $0.29(0.06-1.51)$ & $0.21(0.02-3.00)$ & $4.24(0.34-52.80)$ \\
\hline Stage 1, No & 448 & 4929 & 3069 & 669 & 105 \\
\hline Event, No & 77 & 421 & 187 & 43 & 4 \\
\hline HR $(95 \%$ CI $)$ & $2.15(1.67-2.76)^{* *}$ & Ref. & $0.71(0.60-0.85) *$ & $0.84(0.61-1.15)$ & $0.49(0.18-1.30)$ \\
\hline Stage 2, No & 655 & 4561 & 2957 & 406 & 88 \\
\hline Event, No & 198 & 822 & 303 & 51 & 6 \\
\hline HR $(95 \%$ CI $)$ & $1.95(1.67-2.28)^{* *}$ & Ref. & $0.77(0.68-0.88)^{*}$ & $0.71(0.54-0.95)$ * & $0.40(0.18-0.90)$ * \\
\hline Stage 3, No & 1004 & 8829 & 4468 & 786 & 116 \\
\hline Event, No & 347 & 1970 & 833 & 155 & 20 \\
\hline HR $(95 \%$ CI $)$ & $1.83(1.63-2.05)^{* *}$ & Ref. & $0.83(0.76-0.90)^{* *}$ & $0.91(0.77-1.07)$ & $0.84(0.54-1.31)$ \\
\hline Stage 4 , No & 720 & 5043 & 1908 & 331 & 61 \\
\hline Event, No & 560 & 3687 & 1371 & 233 & 40 \\
\hline HR $(95 \%$ CI $)$ & $1.23(1.13-1.35)^{* *}$ & Ref. & $0.92(0.86-0.98)$ * & $0.90(0.79-1.03)$ & $0.73(0.53-1.00)$ * \\
\hline \multicolumn{6}{|c|}{ Site } \\
\hline $\begin{array}{l}\text { Malignant neoplasm } \\
\text { of colon, No }\end{array}$ & 1847 & 15,293 & 7301 & 1448 & 264 \\
\hline Event, No & 737 & 4449 & 1670 & 308 & 52 \\
\hline HR $(95 \%$ CI $)$ & $1.45(1.34-1.57)^{* *}$ & Ref. & $0.85(0.80-0.90)^{* *}$ & $0.85(0.75-0.95)$ * & $0.79(0.60-1.04)$ \\
\hline $\begin{array}{l}\text { Rectosigmoid } \\
\text { junction, No }\end{array}$ & 234 & 1975 & 1015 & 188 & 29 \\
\hline Event, No & 103 & 587 & 219 & 41 & 2 \\
\hline $\operatorname{HR}(95 \% \mathrm{CI})$ & $1.57(1.26-1.96)^{* *}$ & Ref. & $0.79(0.68-0.93) *$ & $0.82(0.59-1.12)$ & $0.19(0.05-0.77)$ * \\
\hline Rectum, No & 786 & 6507 & 3425 & 610 & 93 \\
\hline Event, No & 346 & 1875 & 807 & 134 & 17 \\
\hline $\operatorname{HR}(95 \% \mathrm{CI})$ & $1.73(1.54-1.95)^{* *}$ & Ref. & $0.86(0.79-0.93) *$ & $0.89(0.75-1.06)$ & $0.65(0.40-1.06)$ \\
\hline
\end{tabular}

\section{Discussion}

Globally, the adult population's BMI has followed an increasing trend over the past 30 years [23]. According to the WHO's statistical data, about 1.5 billion adults are overweight, and 500 million are obese [2]. Obesity is one of the proven risk factors for CRC development and death [24], but only recently has research investigated the effect of obesity on survival following a cancer diagnosis [25]. In other words, its association with oncologic outcomes among CRC patients is ambiguous, and conflicting results have been reported [26].

The mechanisms of the relationship between excess weight and CRC remain unclear. Several possibilities have been hypothesized [8] and primarily linked with obesity-related hormonal changes [14]. The insulin/insulin-like growth factor (IGF) axis and adipokines (adiponectin and leptin) are the two hormonal systems that have been investigated most thoroughly [8]. Obesity correlates with elevated insulin levels, free IGFs, and adipocyte- 
derived factors, including leptin, the tumor necrosis factor-alpha (TNF- $\alpha$ ), and interleukin-6 (IL-6). Besides this, it is also related to adiponectin reduction [27]. These obesity-related hormonal changes have been noted to have a positive association with CRC incidence [28], but because of their interrelations, it has been hard to clarify their causal relationship [14]. However, an increased amount of circulating insulin and free IGF-I has been related to physical inactivity and obesity [29]. Coussens and Werb et al. remarked that obesity-induced chronic inflammation is associated with cancer occurrence [30]. Trevisan et al. also demonstrated that increasing obesity could cause insulin resistance [31], which was reported to have a triple risk of CRC-related mortality in their research involving 62,285 patients [32]. They confirmed that IGF-I and insulin could boost cell proliferation and impede apoptosis in CRC cells [33], suggesting that they may actuate the growth of micro-metastases [26].

However, another mechanism of various BMI levels leading to CRC survival differences is suggested by the evidence of fatty acid synthase (FASN) expression. In normal weight and minimally overweight patients (BMI $<27.5 \mathrm{~kg} / \mathrm{m}^{2}$ ), FASN positivity was noted with a lower mortality rate, whereas among moderately overweight patients (BMI $\geq 27.5 \mathrm{~kg} / \mathrm{m}^{2}$ ), overexpression of FASN increased the mortality rate significantly [34]. FASN essentially leads to de novo lipogenesis and is physiologically adjusted by energy balance. Besides this, both exercise and energy restriction downregulate FASN [35]. This phenomenon has raised the question of whether different severity levels of obesity would lead to conflicting oncologic outcomes during cancer management.

In the context of disease, overweight patients may have survival benefits attributed to a better nutritional status, more optimal medical treatment, more significant endothelial progenitor cells, lower thromboxane production, higher ghrelin sensitivity, and lower concentrations of TNF- $\alpha$ [36]. Overweight CRC patients might have more significant muscle and fat mass, empowering them to deal with tumor progression and the metabolic demands of treatment. However, even though mortality was lowest in the overweight group, the risk was similarly low in high-normal weight patients, suggesting that a BMI in this range may minimize the risks during cancer treatment [36]. In a multivariate analysis by Sinicrope et al. [26], better OS rates in overweight patients were shown. However, this phenomenon was only noted in men $(p=0.006)$ after adjusting for the factors of age, stage, and treatment strategies. Other specialists have also demonstrated that overweight patients with no cancer history have the lowest mortality risk [37,38]. However, this result may reveal a limitation of the BMI analysis because it cannot identify body weight from muscle versus fat; therefore, muscular patients could be categorized as overweight or obese, though they are not really. Of course, these findings that suggest that being overweight is related to a lower mortality rate might not necessarily be causal but rather they reveal that the reference (normal weight) group may include initially overweight patients who might have lost weight due to disease. This would mean increased patient numbers in the reference group with underlying diseases or weight lost due to CRC progression. As their BMI was recorded at the time of cancer diagnosis (BMI at diagnosis), a possible bias related to reverse causation has raised a concern.

Our series found that the higher BMI our patients had, the better OS rates they encountered (Table 2). However, Campbell et al. found a higher relative risk of allcause mortality among patients with class II or III obesity (BMI of $\geq 35$ or $\geq 40 \mathrm{~kg} / \mathrm{m}^{2}$, respectively) [39]. It is essential to realize that one of the leading causes of mortality in these obese CRC patients was cardiovascular disease rather than cancer recurrence, leading to an increased overall mortality. However, Americans are more commonly obese (33.7\%) than the Taiwanese $(7.2 \%)$. Besides this, being very obese is much more common in the American population: $13.4 \%$ of the total U.S. sample and about $1 \%$ in Taiwan are obese. The average BMI values of the obese and very obese are higher in the American group than in the Taiwanese group [40], which might have led to this discrepancy.

In a prior study by Flegal et al. [41], mortality in underweight CRC patients was more often due to non-cancer-related causes than that in normal weight patients. This increased risk of mortality could also be associated with other underlying diseases such 
as advanced type two diabetes [42], cardiac failure [43], and pulmonary diseases [44]. As weight loss is one of the CRC symptoms, more advanced cancer patients would experience more significant weight loss and possibly be underweight at diagnosis [11]. Significant cancer-associated weight loss might identify a poorer prognostic subgroup because cancer cachexia leads to poorer outcomes [45]. The depletion of adipose tissue accounts for most cancer-related weight loss, yet the preferential loss of the skeletal muscle negatively impacts mortality [46].

Our study found that CRC-specific survival rates decreased among underweight patients (Table 2). This phenomenon was encountered after an adjusted analysis with three types of combinations (age with gender, age with gender and stage, and multivariate). These findings emphasize BMI as a decisive prognostic factor for CRC, with the underweight BMI category being disadvantageous for CRC patients. This result has been a consistent finding among the studies mentioned above. Another significant result was that underweight patients had increased cancer recurrence rates (Table 1) and poorer oncologic outcomes in a multivariate analysis (Table 2). The results of shorter OS, DFS, and CRC-specific survival rates for underweight patients suggested that the prognosis was also cancer-related (Table 3 and Figure 2, Table 4 and Figure 3, Table 5 and Figure 4). Our evidence shows that the time frame during which BMI is determined could affect its relationship to the clinical outcome. Since BMI was recorded at diagnosis and enrollment in our study, it is essential to differentiate underweight patients who had a stable weight over time from those who underwent significant cancer-related weight loss at diagnosis. However, some patients would intentionally lose weight through a healthier diet and exercise, whereas other patients experience weight loss because of cancer progression or treatment side effects. Our BMI at diagnosis data cannot clarify these causes of weight loss, which might account for the lack of precise association between BMI at diagnosis and the risk of mortality, which is a limitation of our study.

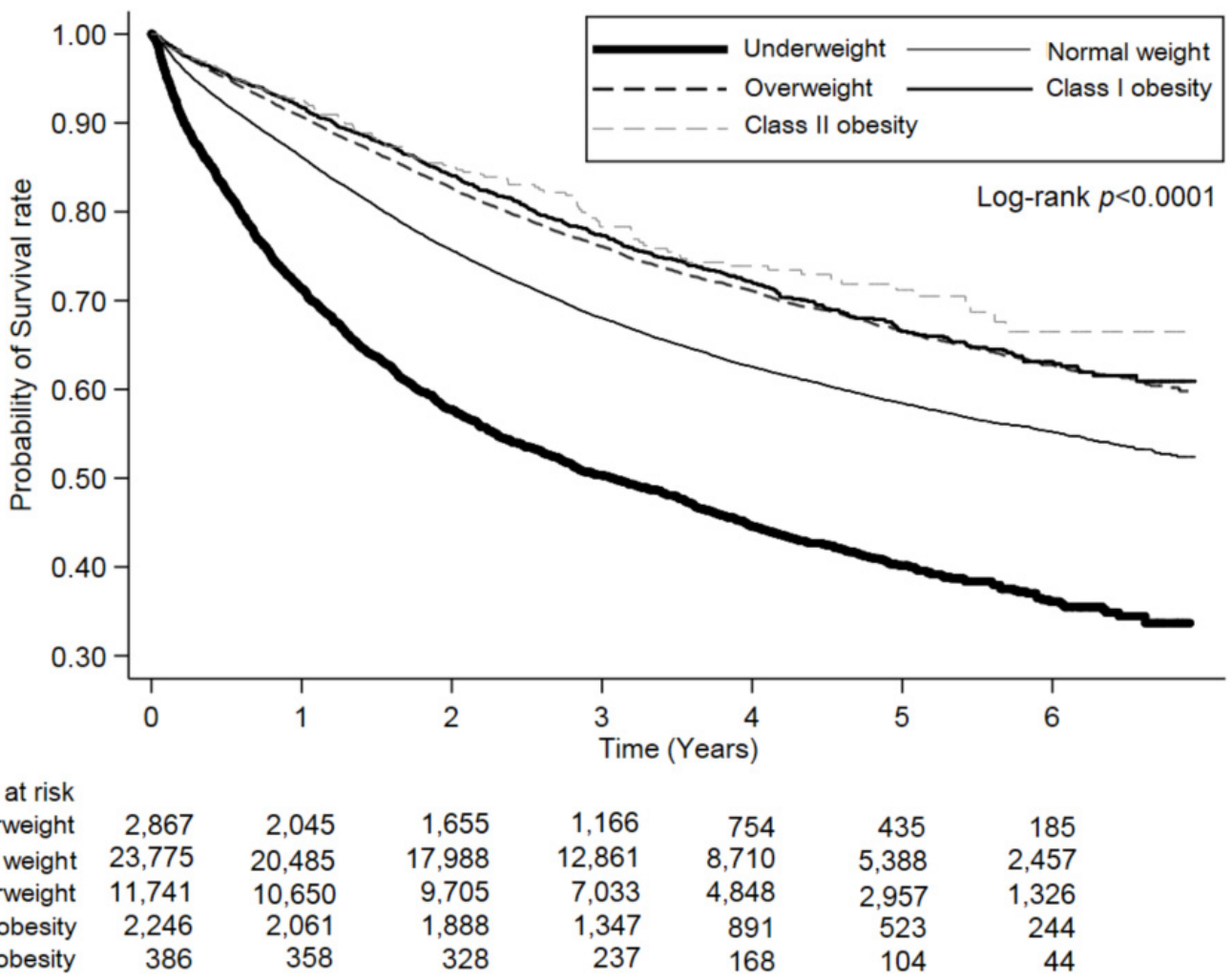

Figure 2. Kaplan-Meier curves for overall survival (OS) rates across the body mass index (BMI) categories. 


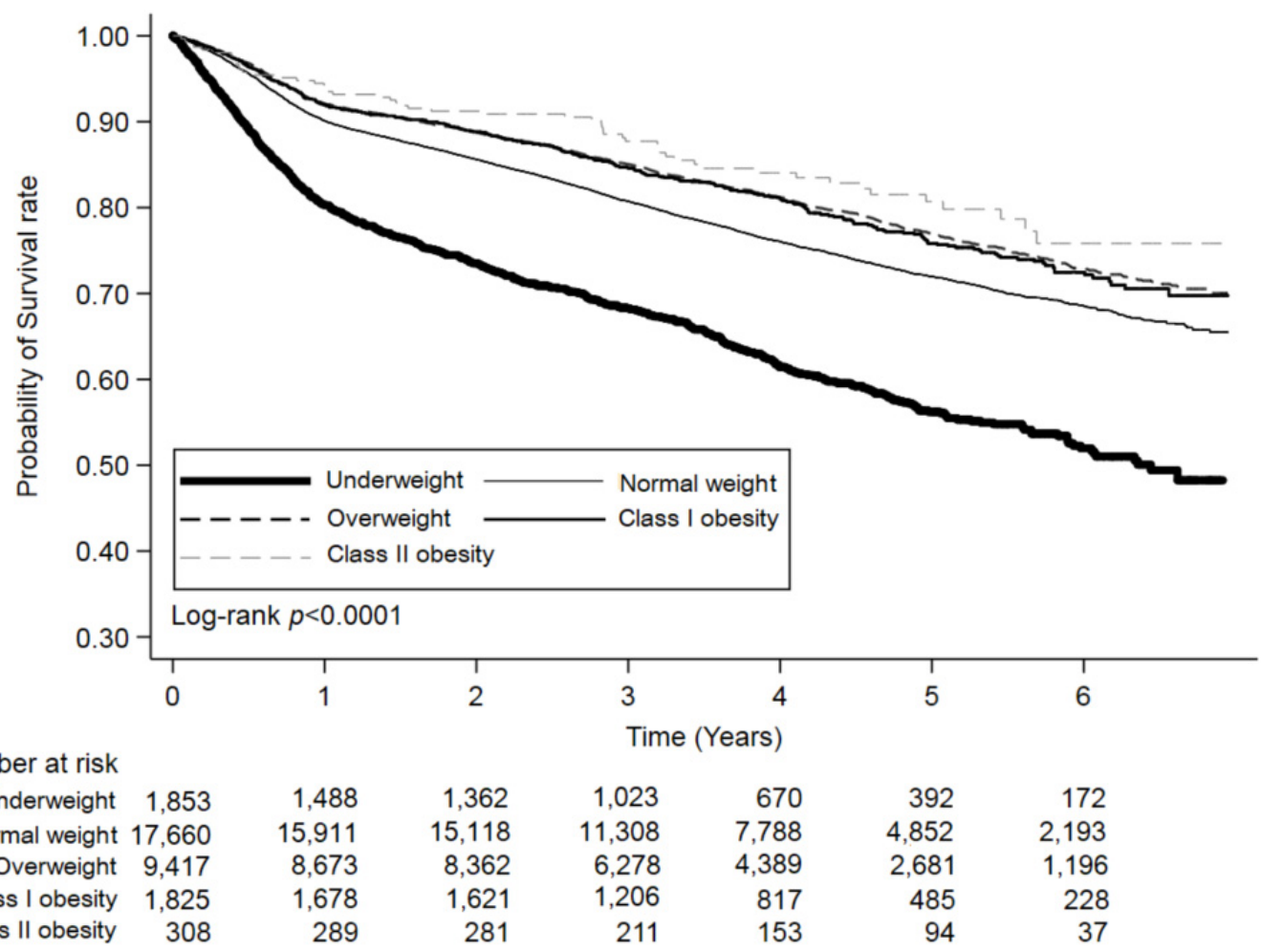

Figure 3. Kaplan-Meier curves for disease-free survival (DFS) rates across the body mass index (BMI) categories.

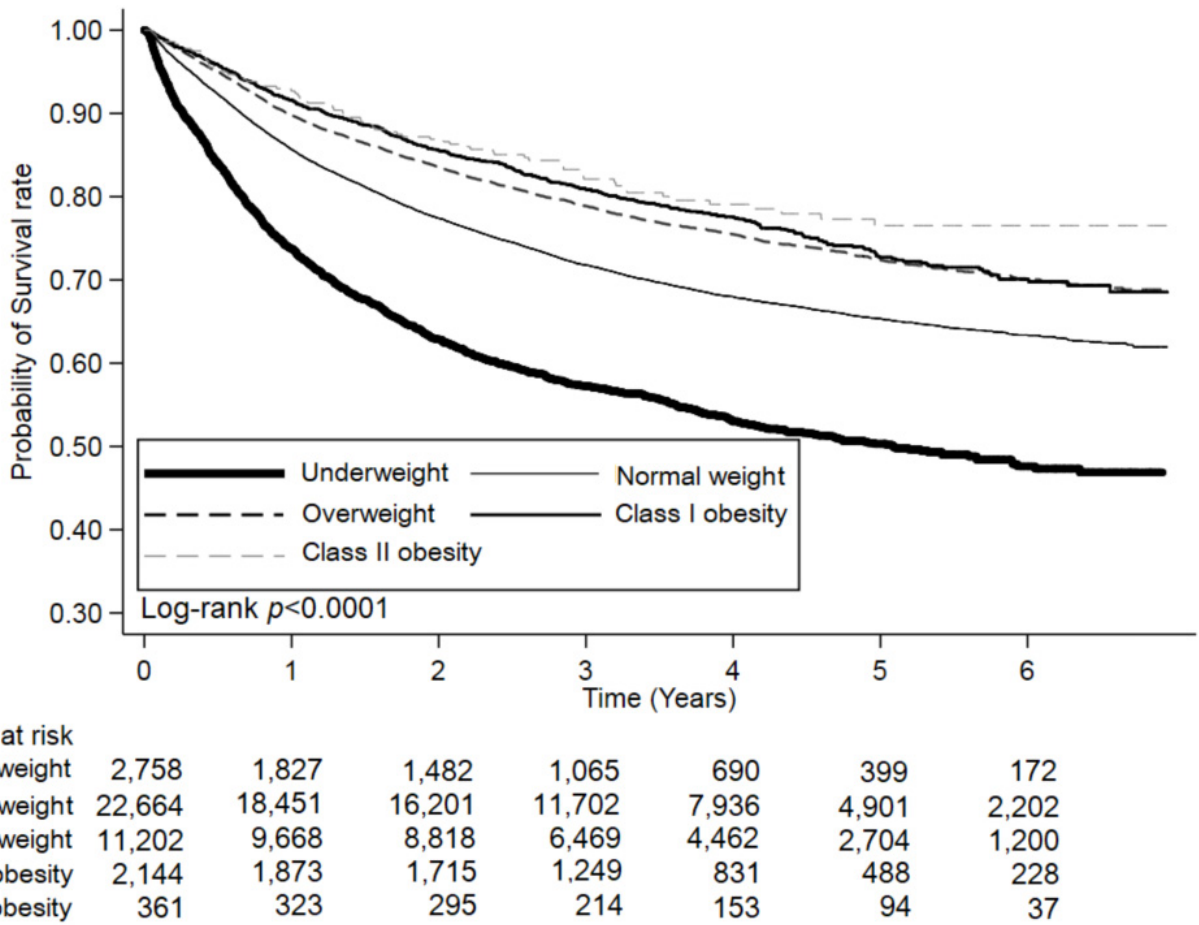

Figure 4. Kaplan-Meier curves for CRC-specific survival rates across the body mass index (BMI) categories.

To date, inconsistent data exist regarding whether the association of BMI with CRCspecific survival rates might differ by patient gender $[25,26,47]$. Polednak et al. performed a meta-analysis involving 153,760 U.S. patients [47]. They showed that the risk ratio of CRC occurrence was 1.4 for obese men and 1.1 for obese women. This also showed that 
this affiliation was weaker for women in comparison with men. Slattery et al. [48] showed that patient gender was related to the colon tumorigenesis molecular pathway in that CRC with defective DNA mismatch repair (MMR) was more prevalent in elderly women but not in men. They also observed that an excess of CRCs with defective MMR in elderly women in their study was related to estrogen withdrawal. Sinicrope et al. [26] also remarked that obese patients had significantly fewer CRCs with defective MMR than normal weight patients did.

Another mechanism regarding this discrepancy may be associated with body fat distribution in that a higher BMI is more commonly associated with a higher ratio of abdominal or central adiposity in men [49]. Abdominal adiposity is closely linked with hyperinsulinemia, insulin resistance, and the IGF axis as potential mediators of increased CRC risk and related mortality [50,51]. The lessened impact of obesity on CRC prognosis observed in women vs. men may be related to estrogen effect modifications. Estrogen levels are correlated with BMI in postmenopausal women because their primary source is androgen conversion in adipose tissue [52]. However, this relationship of obesity with CRC risk is lessened after menopause [53], and hormone replacement therapy (HRT) is consistently related to decreased CRC-related mortality rates [54]. Obesity correlates with increased circulating estrogen levels, and estrogen might protect against CRC development in women with defective MMR [48]. Meanwhile, a profound interaction was noticed between underweight status and patient gender, where underweight men had a 39\% increase in all-cause mortality compared to normal weight men or women [55]. In our results, a similar phenomenon is demonstrated in Table 2.

In a population-based, case-control study, Russo et al. [56] pointed out that both obesity and physical activity were related to a lower prevalence of CRCs with defective MMR in women but not in men. However, the association between physical activity and cancer is debatable. Although 13-14\% of CRC cases may be related to physical inactivity in the U.S. [57], other studies have showed that body weight loss was not sufficient to reduce cancer risk [58]. Physical inactivity deteriorates adipose tissue accumulation, and the subsequent activation of inflammatory cytokines could promote CRC development [59]. Thus, exercise and reducing body fat could alleviate the influence of these factors. A healthier lifestyle, including effective exercise and maintaining a healthy body weight, should be actively promoted to improve a patient's prognosis [60]. However, the issue of physical activity could not be analyzed in our nationwide population database.

Comorbidity is defined as other underlying diseases in addition to an index disease (e.g., CRC) of primary interest [61]. Comorbid conditions in oncologic patients influence the timing of cancer detection, treatment, prognosis, and outcomes [62]. As others have assumed, comorbidity may be partly accountable for the decreased survival rate noticed in African Americans [63]. Since comorbid conditions apply their effects at multiple levels of oncologic patient care, the failure to explain comorbidity in the studies could confound bias [64]. In Table 1, the underweight patients were noted to have a higher ratio of comorbidities, such as myocardial infarction, cardiovascular disease, dementia, rheumatic disease, peptic ulcer disease, hemiplegia, or paraplegia. These all may contribute to their poorer oncologic outcomes and their increased mortality risk.

There were strengths and limitations of our study. The strengths were the sizeable nationwide sample size, the precise record of BMI measurements, the data on treatment and comorbidities, and the accurate collection of data on survival and recurrence over a prolonged follow-up period of 7 years. The limitations included the retrospective design and the sole measurement of obesity by BMI. BMI alone may not contribute sufficiently accurate information to distinguish body fat and lean mass and fat distribution, and these particulars are significantly different based on age, gender, geographic region, and ethnicity. We were also unable to analyze other parameters such as physical activity, diet, menopausal condition, or HRT use, which might be independently associated with the oncologic outcomes and the mortality risk from other causes. Another major limitation was the probability of reverse causation, which might preclude us from determining a pure 
group of healthy normal weight people. We might have underestimated the true impact of obesity on oncologic outcomes, since the advancing cancer condition typically leads to weight loss instead of weight gain. Besides this, BMI pre-diagnosis and at diagnosis are correlated with each other, and it is therefore hard to predict the precise time when obesity is acting. However, since the pre-diagnosis BMI is an essential determinant of the BMI at diagnosis, it is strongly recommended that long-term body weight within the normal range is maintained, rather than counting on weight loss after a cancer diagnosis.

\section{Conclusions}

In conclusion, an underweight BMI is correlated with an increased mortality rate in CRC survivors, particularly in men. In underweight patients, a higher recurrence ratio and reduced rates of DFS, CRC-specific survival, and OS suggest increased tumor aggressiveness. Together, these data lead us to propose that active management to improve an underweight BMI after a CRC diagnosis can improve patient outcomes. Future metaanalysis studies should perform more stratified analyses to investigate various populations and examine the impacts on other possible adjustment factors.

Author Contributions: Conceptualization, C.-C.C. and C.-M.H.; methodology, C.-C.C. and C.-H.H.; software, H.-Y.S.; validation, C.-C.C., Y.-C.W., K.-M.L. and P.-H.L.; acquisition of data, C.-M.H., S.-R.C. and K.-C.C.; formal analysis, C.-C.C., C.-H.H. and H.-Y.S.; investigation, C.-C.C.; resources, J.-J.W., S.-R.C. and K.-C.C.; data curation, C.-H.H.; making tables and graph, C.-H.H.; writing-original draft preparation, C.-C.C.; writing-review and editing, C.-C.C.; visualization, C.-C.L., H.-M.L., C.-M.C. (Chien-Ming Chao) and C.-M.C. (Chin-Ming Chen); supervision, C.-C.C., S.-R.C. and K.-C.C.; funding acquisition, C.-H.H.; obtaining IRB approval, C.-M.C. (Chien-Ming Chao) and C.-M.C. (ChinMing Chen); project administration, L.-R.Y. and T.-C.S.; providing opinion and feedback, C.-M.H., S.-R.C., J.-J.W., P.-H.L. and K.-C.C. All authors have read and agreed to the published version of the manuscript.

Funding: This study was supported by MOHW110-TDU-B-212-144020 from the health and welfare surcharge of tobacco products.

Institutional Review Board Statement: The study was conducted according to the guidelines of the Declaration of Helsinki and approved by the Institutional Review Board of Chi Mei Medical Center, Tainan, Taiwan.

Informed Consent Statement: Patient consent was waived due to non-identification of patient data.

Data Availability Statement: The data sources are the Taiwan Nation Health Insurance Database and Taiwan Cancer Registry. The data are available with the permission from Taiwan Health and Welfare Data Science Centre (https:/ / dep.mohw.gov.tw/DOS/np-2497-113.html, accessed on 5 May 2021). Restrictions apply to the availability of these data, which were used under license for this study.

Acknowledgments: The authors are grateful to Health Data Science Center, National Cheng Kung University Hospital for providing administrative and technical support.

Conflicts of Interest: The funders had no role in the design of the study; in the collection, analyses, or interpretation of data; in the writing of the manuscript; or in the decision to publish the results.

\section{References}

1. Ferlay, J.; Soerjomataram, I.; Dikshit, R.; Eser, S.; Mathers, C.; Rebelo, M.; Parkin, D.M.; Forman, D.; Bray, F. Cancer incidence and mortality worldwide: Sources, methods and major patterns in GLOBOCAN 2012. Int. J. Cancer 2015, 136, E359-E386. [CrossRef] [PubMed]

2. Whitlock, K.; Gill, R.S.; Birch, D.W.; Karmali, S. The Association between Obesity and Colorectal Cancer. Gastroenterol. Res. Pr. 2012, 2012, 1-6. [CrossRef]

3. Sung, J.J.; Lau, J.Y.; Goh, K.; Leung, W.K. Increasing incidence of colorectal cancer in Asia: Implications for screening. Lancet Oncol. 2005, 6, 871-876. [CrossRef]

4. Martinez-Useros, J.; Garcia-Foncillas, J. Obesity and colorectal cancer: Molecular features of adipose tissue. J. Transl. Med. 2016, 14, 1-12. [CrossRef] 
5. Aparicio, T.; Ducreux, M.; Faroux, R.; Barbier, E.; Manfredi, S.; Lecomte, T.; Etienne, P.-L.; Bedenne, L.; Bennouna, J.; Phelip, J.-M.; et al. Overweight is associated to a better prognosis in metastatic colorectal cancer: A pooled analysis of FFCD trials. Eur. J. Cancer 2018, 98, 1-9. [CrossRef]

6. Vrieling, A.; Kampman, E. The role of body mass index, physical activity, and diet in colorectal cancer recurrence and survival: A review of the literature. Am. J. Clin. Nutr. 2010, 92, 471-490. [CrossRef]

7. Harriss, D.J.; Atkinson, G.; George, K.; Cable, N.T.; Reilly, T.; Haboubi, N.; Zwahlen, M.; Egger, M.; Renehan, A. The C-CLEAR group Lifestyle factors and colorectal cancer risk (1): Systematic review and meta-analysis of associations with body mass index. Color. Dis. 2009, 11, 547-563. [CrossRef] [PubMed]

8. Ma, Y.; Yang, Y.; Wang, F.; Zhang, P.; Shi, C.; Zou, Y.; Qin, H. Obesity and Risk of Colorectal Cancer: A Systematic Review of Prospective Studies. PLoS ONE 2013, 8, e53916. [CrossRef] [PubMed]

9. Baade, P.D.; Meng, X.; Youl, P.H.; Aitken, J.; Dunn, J.; Chambers, S.K. The Impact of Body Mass Index and Physical Activity on Mortality among Patients with Colorectal Cancer in Queensland, Australia. Cancer Epidemiol. Biomark. Prev. 2011, 20, 1410-1420. [CrossRef]

10. Kuiper, J.G.; Phipps, A.I.; Neuhouser, M.L.; Chlebowski, R.T.; Thomson, C.A.; Irwin, M.L.; Lane, R.S.; Wactawski-Wende, J.; Hou, L.; Jackson, R.D.; et al. Recreational physical activity, body mass index, and survival in women with colorectal cancer. Cancer Causes Control 2012, 23, 1939-1948. [CrossRef] [PubMed]

11. Meyerhardt, J.A.; Tepper, J.E.; Niedzwiecki, D.; Hollis, D.R.; McCollum, A.D.; Brady, D.; O'Connell, M.J.; Mayer, R.J.; Cum-mings, B.; Willett, C.; et al. Impact of body mass index on outcomes and treatment-related toxicity in patients with stage II and III rectal cancer: Findings from Intergroup Trial 0114. J. Clin. Oncol. 2004, 22, 648-657. [CrossRef]

12. Meyerhardt, J.A.; Catalano, P.J.; Haller, D.G.; Mayer, R.J.; Benson, A.B., 3rd; Macdonald, J.S.; Fuchs, C.S. Influence of body mass index on outcomes and treatment-related toxicity in patients with colon carcinoma. Cancer 2003, 98, 484-495. [CrossRef] [PubMed]

13. Meyerhardt, J.A.; Niedzwiecki, D.; Hollis, D.; Saltz, L.; Mayer, R.J.; Nelson, H.; Whittom, R.; Hantel, A.; Thomas, J.; Fuchs, C.S. Impact of Body Mass Index and Weight Change After Treatment on Cancer Recurrence and Survival in Patients with Stage III Colon Cancer: Findings From Cancer and Leukemia Group B 89803. J. Clin. Oncol. 2008, 26, 4109-4115. [CrossRef]

14. Lee, J.; Meyerhardt, J.A.; Giovannucci, E.; Jeon, J.Y. Association between Body Mass Index and Prognosis of Colorectal Cancer: A Meta-Analysis of Prospective Cohort Studies. PLoS ONE 2015, 10, e0120706. [CrossRef] [PubMed]

15. Ma, R.C.; Chan, J. Type 2 diabetes in East Asians: Similarities and differences with populations in Europe and the United States. Ann. N. Y. Acad. Sci. 2013, 1281, 64-91. [CrossRef] [PubMed]

16. Min, Y.W.; Kim, S.A.; Lee, J.H.; Kim, J.Y.; Chang, D.K.; Rhee, P.L.; Kim, J.J.; Rhee, J.C.; Kim, Y.H. Overweight is associated with a favorable survival in patients with colorectal cancer: A prospective cohort study in an Asian population. Ann. Surg. Oncol. 2012, 19, 3460-3464. [CrossRef] [PubMed]

17. Yamamoto, N.; Fujii, S.; Sato, T.; Oshima, T.; Rino, Y.; Kunisaki, C.; Masuda, M.; Imada, T. Impact of body mass index and visceral adiposity on outcomes in colorectal cancer. Asia-Pac. J. Clin. Oncol. 2012, 8, 337-345. [CrossRef]

18. Liu, D.; Li, Q.; Yang, Z.; Hu, X.; Qian, W.; Du, Y.; Liu, B. Association of body mass index and smoking on outcome of Chinese patients with colorectal cancer. World J. Surg. Oncol. 2013, 11, 271. [CrossRef]

19. Moon, H.-G.; Ju, Y.-T.; Jeong, C.-Y.; Jung, E.-J.; Lee, Y.-J.; Hong, S.-C.; Ha, W.-S.; Park, S.-T.; Choi, S.-K. Visceral Obesity May Affect Oncologic Outcome in Patients with Colorectal Cancer. Ann. Surg. Oncol. 2008, 15, 1918-1922. [CrossRef] [PubMed]

20. Wang, N.; Khankari, N.K.; Cai, H.; Li, H.-L.; Yang, G.; Gao, Y.-T.; Xiang, Y.-B.; Shu, X.-O.; Zheng, W. Prediagnosis body mass index and waist-hip circumference ratio in association with colorectal cancer survival. Int. J. Cancer 2016, 140, 292-301. [CrossRef]

21. Ho, C.-H.; Cheng, K.-C.; Chao, C.-M.; Lai, C.-C.; Chiang, S.-R.; Chen, C.-M.; Liao, K.-M.; Wang, J.-J.; Lee, P.-H.; Hung, C.-M.; et al. Does radiotherapy increase the risk of colorectal cancer among prostate cancer patients? A large population-based study. $J$. Cancer 2020, 11, 6204-6212. [CrossRef]

22. Daniel, C.R.; Shu, X.; Ye, Y.; Gu, J.; Raju, G.S.; Kopetz, S.; Wu, X. Severe obesity prior to diagnosis limits survival in colorectal cancer patients evaluated at a large cancer centre. Br. J. Cancer 2015, 114, 103-109. [CrossRef] [PubMed]

23. Finucane, M.M.; Stevens, G.A.; Cowan, M.J.; Danaei, G.; Lin, J.K.; Paciorek, C.J.; Singh, G.M.; Gutierrez, H.R.; Lu, Y.; Bahalim, A.N.; et al. National, regional, and global trends in body-mass index since 1980: Systematic analysis of health examination surveys and epidemiological studies with 960 country-years and 9.1 million participants. Lancet 2011, 377, 557-567. [CrossRef]

24. Larsson, S.C.; Wolk, A. Obesity and colon and rectal cancer risk: A meta-analysis of prospective studies. Am. J. Clin. Nutr. 2007, 86, 556-565. [CrossRef] [PubMed]

25. Hines, R.B.; Shanmugam, C.; Waterbor, J.W.; McGwin, G.; Funkhouser, E.; Coffey, C.S.; Posey, J.; Manne, U. Effect of Comorbidity and Body Mass Index on Colon Cancer Survival of African American and Caucasian Patients. Cancer 2009, 115, 5798-5806. [CrossRef] [PubMed]

26. Sinicrope, F.A.; Foster, N.R.; Sargent, D.; O'Connell, M.J.; Rankin, C. Obesity Is an Independent Prognostic Variable in Colon Cancer Survivors. Clin. Cancer Res. 2010, 16, 1884-1893. [CrossRef]

27. Renehan, A.G.; Roberts, D.; Dive, C. Obesity and cancer: Pathophysiological and biological mechanisms. Arch. Physiol. Biochem. 2008, 114, 71-83. [CrossRef] [PubMed] 
28. Larsson, S.C.; Orsini, N.; Wolk, A. Diabetes Mellitus and Risk of Colorectal Cancer: A Meta-Analysis. J. Natl. Cancer Inst. 2005, 97, 1679-1687. [CrossRef]

29. Merkow, R.P.; Bilimoria, K.Y.; McCarter, M.D.; Bentrem, D.J. Effect of Body Mass Index on Short-Term Outcomes after Colectomy for Cancer. J. Am. Coll. Surg. 2009, 208, 53-61. [CrossRef]

30. Coussens, L.M.; Werb, Z. Inflammation and cancer. Nature 2002, 420, 860-867. [CrossRef]

31. Ashrafian, H.; Ahmed, K.; Rowland, S.P.; Patel, V.M.; Gooderham, N.J.; Holmes, E.; Darzi, A.; Athanasiou, T. Metabolic surgery and cancer: Protective effects of bariatric procedures. Cancer 2011, 117, 1788-1799. [CrossRef]

32. Trevisan, M.; Liu, J.; Muti, P.; Misciagna, G.; Menotti, A.; Fucci, F.; Trevisan, M.; Liu, J.; Muti, P.; Misciagna, G.; et al. Markers of insulin resistance and colorectal cancer mortality. Cancer Epidemiol. Biomark. Prev. 2001, 10, 937-941.

33. Guo, Y.S.; Narayan, S.; Yallampalli, C.; Singh, P. Characterization of insulin like growth factor I receptors in human colon cancer. Gastroenterology 1992, 102, 1101-1108. [CrossRef]

34. Ogino, S.; Nosho, K.; Meyerhardt, J.A.; Kirkner, G.J.; Chan, A.T.; Kawasaki, T.; Giovannucci, E.L.; Loda, M.; Fuchs, C.S. Cohort Study of Fatty Acid Synthase Expression and Patient Survival in Colon Cancer. J. Clin. Oncol. 2008, 26, 5713-5720. [CrossRef] [PubMed]

35. Menendez, J.; Lupu, R. Fatty acid synthase and the lipogenic phenotype in cancer pathogenesis. Nat. Rev. Cancer 2007, 7, $763-777$. [CrossRef]

36. Kroenke, C.H.; Neugebauer, R.; Meyerhardt, J.; Prado, C.M.; Weltzien, E.; Kwan, M.L.; Xiao, J.; Caan, B. Analysis of Body Mass Index and Mortality in Patients with Colorectal Cancer Using Causal Diagrams. JAMA Oncol. 2016, 2, 1137-1145. [CrossRef]

37. Flegal, K.M.; Graubard, B.I.; Williamson, D.F.; Gail, M.H. Cause-Specific Excess Deaths Associated with Underweight, Overweight, and Obesity. JAMA 2007, 298, 2028-2037. [CrossRef] [PubMed]

38. Orpana, H.M.; Berthelot, J.M.; Kaplan, M.S.; Feeny, D.H.; McFarland, B.; Ross, N.A. BMI and mortality: Results from a national longitudinal study of Canadian adults. Obesity 2010, 18, 214-218. [CrossRef] [PubMed]

39. Campbell, P.T.; Newton, C.C.; Dehal, A.N.; Jacobs, E.J.; Patel, A.V.; Gapstur, S.M. Impact of Body Mass Index on Survival After Colorectal Cancer Diagnosis: The Cancer Prevention Study-II Nutrition Cohort. J. Clin. Oncol. 2012, 30, 42-52. [CrossRef] [PubMed]

40. Vasunilashorn, S.; Kim, J.K.; Crimmins, E.M. International differences in the links between obesity and physiological dysregulation: The United States, England, and Taiwan. J. Obes. 2013, 2013, 618056. [CrossRef] [PubMed]

41. Dignam, J.J.; Polite, B.N.; Yothers, G.; Raich, P.; Colangelo, L.; O'Connell, M.J.; Wolmark, N. Body Mass Index and Outcomes in Patients Who Receive Adjuvant Chemotherapy for Colon Cancer. J. Natl. Cancer Inst. 2006, 98, 1647-1654. [CrossRef]

42. Sairenchi, T.; Iso, H.; Irie, F.; Fukasawa, N.; Ota, H.; Muto, T. Underweight as a Predictor of Diabetes in Older Adults: A large cohort study. Diabetes Care 2007, 31, 583-584. [CrossRef]

43. Kenchaiah, S.; Pocock, S.J.; Wang, D.; Finn, P.V.; Zornoff, L.A.M.; Skali, H.; Pfeffer, M.A.; Yusuf, S.; Swedberg, K.; Michelson, E.L.; et al. Body mass index and prognosis in patients with chronic heart failure: Insights from the Candesartan in Heart failure: Assessment of Reduction in Mortality and morbidity (CHARM) program. Circulation 2007, 116, 627-636. [CrossRef]

44. Cao, C.; Wang, R.; Wang, J.; Bunjhoo, H.; Xu, Y.; Xiong, W. Body Mass Index and Mortality in Chronic Obstructive Pulmonary Disease: A Meta-Analysis. PLoS ONE 2012, 7, e43892. [CrossRef] [PubMed]

45. Van Vledder, M.G.; Levolger, S.; Ayez, N.; Verhoef, C.; Tran, T.C.K.; Ijzermans, J.N.M. Body composition and outcome in patients undergoing resection of colorectal liver metastases. BJS 2012, 99, 550-557. [CrossRef]

46. Fearon, K.; Strasser, F.; Anker, S.D.; Bosaeus, I.; Bruera, E.; Fainsinger, R.L.; Jatoi, A.; Loprinzi, C.; MacDonald, N.; Mantovani, G.; et al. Definition and classification of cancer cachexia: An international consensus. Lancet Oncol. 2011, 12, 489-495. [CrossRef]

47. Polednak, A.P. Estimating the number of U.S. incident cancers attributable to obesity and the impact on temporal trends in incidence rates for obesity-related cancers. Cancer Detect. Prev. 2008, 32, 190-199. [CrossRef] [PubMed]

48. Slattery, M.L.; Potter, J.; Curtin, K.; Edwards, S.; Ma, K.N.; Anderson, K.; Schaffer, D.; Samowitz, W.S. Estrogens reduce and withdrawal of estrogens increase risk of microsatellite instability-positive colon cancer. Cancer Res. 2001, 61, 126-130.

49. Power, M.L.; Schulkin, J. Sex differences in fat storage, fat metabolism, and the health risks from obesity: Possible evolutionary origins. Br. J. Nutr. 2008, 99, 931-940. [CrossRef]

50. Giovannucci, E. Metabolic syndrome, hyperinsulinemia, and colon cancer: A review. Am. J. Clin. Nutr. 2007, 86, 836S-842S. [CrossRef]

51. Wolpin, B.M.; Meyerhardt, J.A.; Chan, A.T.; Ng, K.; Chan, J.A.; Wu, K.; Pollak, M.N.; Giovannucci, E.L.; Fuchs, C.S. Insulin, the insulin-like growth factor axis, and mortality in patients with nonmetastatic colorectal cancer. J. Clin. Oncol. 2009, 27, 176-185. [CrossRef] [PubMed]

52. McTiernan, A.; Wu, L.; Chen, C.; Chlebowski, R.; Mossavar-Rahmani, Y.; Modugno, F.; Perri, M.G.; Stanczyk, F.Z.; Van Horn, L.; Wang, C. Relation of BMI and Physical Activity to Sex Hormones in Postmenopausal Women*. Obesity 2006, 14, 1662-1677. [CrossRef] [PubMed]

53. Terry, P.D.; Miller, A.B.; Rohan, T.E. Obesity and colorectal cancer risk in women. Gut 2002, 51, 191-194. [CrossRef] [PubMed] 
54. Henderson, K.D.; Duan, L.; Sullivan-Halley, J.; Ma, H.; Clarke, C.A.; Neuhausen, S.L.; Templeman, C.; Bernstein, L. Meno-pausal hormone therapy use and risk of invasive colon cancer: The California Teachers Study. Am. J. Epidemiol. 2010, 171, 415-425. [CrossRef]

55. Sinicrope, F.A.; Foster, N.R.; Yothers, G.; Benson, A.; Seitz, J.F.; Labianca, R.; Goldberg, R.M.; Degramont, A.; O'Connell, M.J.; Sargent, D.J.; et al. Body mass index at diagnosis and survival among colon cancer patients enrolled in clinical trials of adjuvant chemotherapy. Cancer 2013, 119, 1528-1536. [CrossRef]

56. Russo, A.G.; Franceschi, S.; La Vecchia, C.; Maso, L.D.; Montella, M.; Conti, E.; Giacosa, A.; Falcini, F.; Negri, E. Body size and colorectal-cancer risk. Int. J. Cancer 1998, 78, 161-165. [CrossRef]

57. Slattery, M.L.; Berry, T.D.; Potter, J.; Caan, B. Diet diversity, diet composition, and risk of colon cancer (United States). Cancer Causes Control. 1997, 8, 872-882. [CrossRef]

58. Franco, M.; Bilal, U.; Orduñez, P.; Benet, M.; Morejón, A.; Caballero, B.; Kennelly, J.F.; Cooper, R.S. Population-wide weight loss and regain in relation to diabetes burden and cardiovascular mortality in Cuba 1980-2010: Repeated cross sectional surveys and ecological comparison of secular trends. BMJ 2013, 346, f1515. [CrossRef]

59. Volkova, E.; Willis, J.; Wells, J.E.; Robinson, B.; Dachs, G.U.; Currie, M.J. Association of angiopoietin-2, C-reactive protein and markers of obesity and insulin resistance with survival outcome in colorectal cancer. Br. J. Cancer 2010, 104, 51-59. [CrossRef]

60. Jeon, J.Y.; Jeong, D.H.; Park, M.G.; Lee, J.-W.; Chu, S.H.; Park, J.-H.; Lee, M.K.; Sato, K.; Ligibel, J.A.; Meyerhardt, J.A.; et al. Impact of Diabetes on Oncologic Outcome of Colorectal Cancer Patients: Colon vs. Rectal Cancer. PLoS ONE 2013, 8, e55196. [CrossRef]

61. Feinstein, A.R. The pre-therapeutic classification of co-morbidity in chronic disease. J. Chronic Dis. 1970, 23, 455-468. [CrossRef]

62. De Groot, V.; Beckerman, H.; Lankhorst, G.J.; Bouter, L. How to measure comorbidity: A critical review of available methods. J. Clin. Epidemiol. 2003, 56, 221-229. [CrossRef]

63. Polite, B.N.; Dignam, J.J.; Olopade, O.I. Colorectal Cancer Model of Health Disparities: Understanding Mortality Differences in Minority Populations. J. Clin. Oncol. 2006, 24, 2179-2187. [CrossRef] [PubMed]

64. Extermann, M. Measuring comorbidity in older cancer patients. Eur. J. Cancer 2000, 36, 453-471. [CrossRef] 